\title{
Lower bounds on the dilation of plane spanners*
}

\author{
Adrian Dumitrescu \\ Department of Computer Science \\ University of Wisconsin-Milwaukee \\ Milwaukee, WI, USA \\ dumitres@uwm.edu
}

\author{
Anirban Ghosh \\ Department of Computer Science \\ University of Wisconsin-Milwaukee \\ Milwaukee, WI, USA \\ anirban@uwm.edu
}

July 30, 2018

\begin{abstract}
(I) We exhibit a set of 23 points in the plane that has dilation at least 1.4308, improving the previous best lower bound of 1.4161 for the worst-case dilation of plane spanners.

(II) For every $n \geq 13$, there exists an $n$-element point set $S$ such that the degree 3 dilation of $S$ equals $1+\sqrt{3}=2.7321 \ldots$ in the domain of plane geometric spanners. In the same domain, we show that for every $n \geq 6$, there exists a an $n$-element point set $S$ such that the degree 4 dilation of $S$ equals $1+\sqrt{(5-\sqrt{5}) / 2}=2.1755 \ldots$ The previous best lower bound of 1.4161 holds for any degree.

(III) For every $n \geq 6$, there exists an $n$-element point set $S$ such that the stretch factor of the greedy triangulation of $S$ is at least 2.0268 .
\end{abstract}

Keywords: geometric graph, plane spanner, vertex dilation, stretch factor.

\section{Introduction}

Given a set of points $P$ in the Euclidean plane, a geometric graph on $P$ is a weighted graph $G=(V, E)$ where $V=P$ and an edge $u v \in E$ is the line segment with endpoints $u, v \in V$ weighted by the Euclidean distance $|u v|$ between them. For $t \geq 1$, a geometric graph $G$ is a $t$-spanner, if for every pair of vertices $u, v$ in $V$, the length of the shortest path $\pi_{G}(u, v)$ between them in $G$ is at most $t$ times $|u v|$, i.e., $\forall u, v \in V,\left|\pi_{G}(u, v)\right| \leq t|u v|$. A complete geometric graph on a set of points is a 1-spanner. Where there is no necessity to specify $t$, we use the term geometric spanner. A geometric spanner $G$ is plane if no two edges in $G$ cross. In this paper we only consider plane geometric spanners. A geometric spanner of degree at most $k$ is referred to as a degree $k$ geometric spanner.

Given a geometric spanner $G=(V, E)$, the vertex dilation or stretch factor of $u, v \in V$, denoted $\delta_{G}(u, v)$, is defined as $\delta_{G}(u, v)=\left|\pi_{G}(u, v)\right| /|u v|$. When $G$ is clear from the context, we simply write $\delta(u, v)$. The vertex dilation or stretch factor of $G$, denoted $\delta(G)$, is defined as $\delta(G)=\sup _{u, v \in V} \delta_{G}(u, v)$. The terms graph theoretic dilation and spanning ratio are also used in the literature. Refer to [22, 29, 35] for such definitions.

Given a point set $P$, let the dilation of $P$, denoted by $\delta_{0}(P)$, be the minimum stretch factor of a plane geometric graph (equivalently, triangulation) on vertex set $P$; see [34. Similarly, let the degree $k$ dilation of $P$, denoted by $\delta_{0}(P, k)$, be the minimum stretch factor of a plane geometric

${ }^{*}$ A preliminary version in: Proceedings of the 2nd International Conference on Algorithms and Discrete Applied Mathematics, Thiruvananthapuram, India, Feb. 2016, vol. 9602 of LNCS. 
graph of degree at most $k$ on vertex set $P$. Clearly, $\delta_{0}(P, k) \geq \delta_{0}(P)$ holds for any $k$. Furthermore, $\delta_{0}(P, j) \geq \delta_{0}(P, k)$ holds for any $j<k$. (Note that the term dilation has been also used with different meanings in the literature, see for instance [11, 30].)

In the last few decades, great progress has been made in the field of geometric spanners; for an overview refer to [25, 35]. Common goals include constructions of low stretch factor geometric spanners that have few edges, bounded degree and so on. A survey of open problems in this area along with existing results can be found in [11. Geometric spanners find their applications in the areas of robotics, computer networks, distributed systems and many others. Refer to [1, 2, 4, 13, 23, 32 for various algorithmic results.

The existence of plane $t$-spanners for some constant $t>1$ (with no restriction on degree) was first investigated by Chew [15] in the 80s. He showed that it is always possible to construct a plane 2-spanner with $O(n)$ edges on a set of $n$ points; he also observed that every plane geometric graph embedded on the 4 points placed at the vertices of a square has stretch factor at least $\sqrt{2}$. This was the best lower bound on the worst-case dilation of plane spanners for almost 20 years until it was shown by Mulzer 34] using a computer program that every triangulation of a regular 21-gon has stretch factor at least $\left(2 \sin \frac{\pi}{21}+\sin \frac{5 \pi}{21}+\sin \frac{3 \pi}{21}\right) / \sin \frac{10 \pi}{21}=1.4161 \ldots$ Henceforth, it was posed as an open problem by Bose and Smid [11, Open Problem 1] (as well as by Kanj in his survey [26, Open Problem 5]): "What is the best lower bound on the spanning ratio of plane geometric graphs? Specifically, is there a $t>\sqrt{2.005367532} \approx 1.41611 \ldots$ and a point set $P$, such that every triangulation of $P$ has spanning ratio at least $t$ ?". We give a positive answer to the second question by showing that a set $S$ of 23 points placed at the vertices of a regular 23-gon, has dilation $\delta_{0}(S) \geq\left(2 \sin \frac{2 \pi}{23}+\sin \frac{8 \pi}{23}\right) / \sin \frac{11 \pi}{23}=1.4308 \ldots$

The problem can be traced back to a survey written by Eppstein [24, Open Problem 9]: "What is the worst case dilation of the minimum dilation triangulation?". The point set $S$ also provides a partial answer for this question. From the other direction, the current best upper bound of 1.998 was proved by Xia [37] using Delaunay triangulations. Note that this bound is only slightly better than the bound of 2 obtained by Chew [15] in the 1980s. For previous results on the upper bound refer to [16, 18, 19, 29].

The design of low degree plane spanners is of great interest to geometers. Bose et al. [9] were the first to show that there always exists a plane $t$-spanner of degree at most 27 on any set of points in the Euclidean plane where $t \approx 10.02$. The result was subsequently improved in [5, 6, 7, 12, 27, 33. in terms of degree. Recently, Kanj et al. [28] showed that $t=20$ can be achieved with degree 4 . However, the question whether the degree can be reduced to 3 remains open at the time of this writing. If one does not insist on having a plane spanner, Das et al. [17] showed that degree 3 is achievable. While numerous papers have focused on upper bounds on the dilation of bounded degree plane spanners, not much is known about lower bounds. In this paper, we explore this direction and provide new lower bounds for unrestricted degrees and when degrees 3 and 4 are imposed.

A greedy triangulation of a finite point set $P$ is constructed in the following way: starting with an empty set of edges $E$, repeatedly add edges to $E$ in non-decreasing order of length as long as edges in $E$ are noncrossing. Bose et al. [10] have showed that the greedy triangulation is a $t$-spanner, where $t=8(\pi-\alpha)^{2} /\left(\alpha^{2} \sin ^{2}(\alpha / 4)\right) \approx 11739.1$ and $\alpha=\pi / 6$. Here we obtain a worst-case lower bound of 2.0268; in light of computational experiments we carried out, we believe that the aforementioned upper bound is very far from the truth.

Related work. If $S_{n}$ is the set of $n$ vertices of a regular $n$-gon, Mulzer 34] showed that $1.3836 \ldots=\sqrt{2-\sqrt{3}}+\sqrt{3} / 2 \leq \delta_{0}\left(S_{n}\right) \leq 0.471 \pi / \sin 0.471 \pi=1.4858 \ldots$, for every $n \geq 74$; the upper bound holds for every $n \geq 3$. Amarnadh and Mitra [3] have shown that in the case of a cyclic 
polygon (a polygon whose vertices are co-circular), the stretch factor of any fan triangulation (i.e., with a vertex of degree $n-1)$ is $\leq 1.4846$.

As mentioned earlier, low degree plane spanners for general point sets have been studied in [5, 7, 9, 12, 27, 33. The construction of low degree plane spanners for the infinite square and hexagonal lattices has been recently investigated in [21].

Bose et al. [8] presented a finite convex point set for which there is a Delaunay triangulation whose stretch factor is at least $1.581>\pi / 2$, thereby disproving a widely believed $\pi / 2$ upper bound conjectured by Chew [15]. They also showed that this lower bound can be slightly raised to 1.5846 if the point set need not be convex. This lower bound for non-convex point sets has been further improved to 1.5932 by Xia and Zhang [38].

Klein et al. [30] proved the following interesting structural result. Let $S$ be a finite set of points in the plane. Then either $S$ is a subset of one of the well-known sets of points whose triangulation is unique and has dilation 1 , or there exists a number $\Delta(S)>1$ such that each finite plane graph containing $S$ among its vertices has dilation at least $\Delta(S)$.

Cheong et al. 14 showed that for every $n \geq 5$, there are sets of $n$ points in the plane that do not have a minimum-dilation spanning tree without edge crossings and that 5 is minimal with this property. They also showed that given a set $S$ of $n$ points with integer coordinates in the plane and a rational dilation $t>1$, it is NP-hard to decide whether a spanning tree of $S$ with dilation at most $t$ exists, regardless if edge crossings are allowed or not.

Knauer and Mulzer [31] showed that for each edge $e$ of a minimum dilation triangulation of a point set, at least one of the two half-disks of diameter about $0.2|e|$ on each side of $e$ and centered at the midpoint of $e$ must be empty of point: 1 .

When the stretch factor (or dilation) is measured over all pairs of points on edges or vertices of a plane graph $G$ (rather than only over pairs of vertices) one arrives at the concept of geometric dilation of $G$; see $[20,22]$.

Our results. (I) Let $S$ be a set of 23 points placed at the vertices of a regular 23-gon. Then, $\delta_{0}(S)=\left(2 \sin \frac{2 \pi}{23}+\sin \frac{8 \pi}{23}\right) / \sin \frac{11 \pi}{23}=1.4308 \ldots$ (Theorem 1 . Section 2). This improves the previous bound of $\left(2 \sin \frac{\pi}{21}+\sin \frac{5 \pi}{21}+\sin \frac{3 \pi}{21}\right) / \sin \frac{10 \pi}{21}=1.4161 \ldots$, due to Mulzer [34], on the worst case dilation of plane spanners.

(II) (a) For every $n \geq 13$, there exists a set $S$ of $n$ points such that $\delta_{0}(S, 3) \geq 1+\sqrt{3}=$ $2.7321 \ldots$ (Theorem 2, Section 3). (b) For every $n \geq 6$, there exists a set $S$ of $n$ points such that $\delta_{0}(S, 4) \geq 1+\sqrt{(5-\sqrt{5}) / 2}=2.1755 \ldots$ (Theorem 3. Section 3). The previous best lower bound of $\left(2 \sin \frac{\pi}{21}+\sin \frac{5 \pi}{21}+\sin \frac{3 \pi}{21}\right) / \sin \frac{10 \pi}{21}=1.4161 \ldots$, due to Mulzer [34] holds for any degree. Here we sharpen it for degrees 3 and 4 .

(III) For every $n \geq 6$, there exists a set $S$ of $n$ points such that the stretch factor of the greedy triangulation of $S$ is at least 2.0268 .

Notations and assumptions. Let $P$ be a planar point set and $G=(V, E)$ be a plane geometric graph on vertex set $P$. For $p, q \in P, p q$ denotes the connecting segment and $|p q|$ denotes its Euclidean length. The degree of a vertex (point) $p \in P$ is denoted by $\operatorname{deg}(p)$. For a specific point set $P=\left\{p_{1}, \ldots, p_{n}\right\}$, we denote a path in $G$ consisting of vertices in the order $p_{i}, p_{j}, p_{k}, \ldots$ using $\rho(i, j, k, \ldots)$ and by $|\rho(i, j, k, \ldots)|$ its total Euclidean length. The graphs we construct have the property that no edge contains a point of $P$ in its interior.

\footnotetext{
${ }^{1}$ Their result inaccurately states that the entire disk of that diameter is an exclusion region.
} 


\section{A new lower bound on the dilation of plane spanners}

In this section, we show that the set $S=\left\{s_{0}, \ldots, s_{22}\right\}$ of 23 points placed at the vertices of a regular 23-gon has dilation $\delta_{0}(S) \geq\left(2 \sin \frac{2 \pi}{23}+\sin \frac{8 \pi}{23}\right) / \sin \frac{11 \pi}{23}=1.4308 \ldots$ (see Fig. 1). Assume that the points lie on a circle of unit radius. We first present a theoretical proof showing that $\delta_{0}(S) \geq\left(\sin \frac{2 \pi}{23}+\right.$ $\left.\sin \frac{4 \pi}{23}+\sin \frac{5 \pi}{23}\right) / \sin \frac{11 \pi}{23}=1.4237 \ldots$; we then raise the bound to $\delta_{0}(S) \geq\left(2 \sin \frac{2 \pi}{23}+\sin \frac{8 \pi}{23}\right) / \sin \frac{11 \pi}{23}=$ $1.4308 \ldots$ using a computer program. The result obtained by the program is tight as there exists a triangulation of $S$ (see Fig. 1 (right)) with stretch factor exactly $\left(2 \sin \frac{2 \pi}{23}+\sin \frac{8 \pi}{23}\right) / \sin \frac{11 \pi}{23}=$ $1.4308 \ldots$

Define the convex hull length of a chord $s_{i} s_{j} \in S$ as $\mu(i, j)=\min (|i-j|, 23-|i-j|)$. Observe that $1 \leq \mu(i, j) \leq 11$. Since triangulations are maximal planar graphs, we only consider triangulations of $S$ while computing $\delta_{0}(S)$; in particular, every edge of the convex hull of $S$ is present. Note that there are $C_{21}=24,466,267,020$ triangulations of $S$. Here $C_{n}=\frac{1}{n+1}\left(\begin{array}{c}2 n \\ n\end{array}\right)$ is the $n^{\text {th }}$ Catalan number and there are $C_{n}$ ways to triangulate a convex polygon with $n+2$ vertices.

If $s_{i}, s_{j} \in S$, then $\left|s_{i} s_{j}\right|=2 \sin \frac{\mu(i, j) \pi}{23}$. Consider a shortest path connecting $s_{i}, s_{j} \in S$ consisting of $k$ edges with convex hull lengths $n_{1}, \ldots, n_{k}$; its length is $|\rho(i, \ldots, j)|=2 \sum_{h=1}^{k} \sin \frac{n_{h} \pi}{23}$. Let $\lambda=\mu(i, j)$ and

$$
g\left(\lambda, n_{1}, \ldots, n_{k}\right)=\frac{|\rho(i, \ldots, j)|}{\left|s_{i} s_{j}\right|}=\frac{\sum_{h=1}^{k} \sin \frac{n_{h} \pi}{23}}{\sin \frac{\lambda \pi}{23}} .
$$

We will use $\lambda=11$ in all subsequent proofs of this section and therefore we set

$$
f\left(n_{1}, \ldots, n_{k}\right):=g\left(11, n_{1}, \ldots, n_{k}\right) .
$$

Various values of $f$, as given by (1) and (2), will be repeatedly used in lower-bounding the stretch factor of point pairs in specific configurations, i.e., when some edges are assumed to be present. Observe that $f$ is a symmetric function that can be easily computed (tabulated) at each tuple $n_{1}, \ldots, n_{k} ;$ see Table 1 .
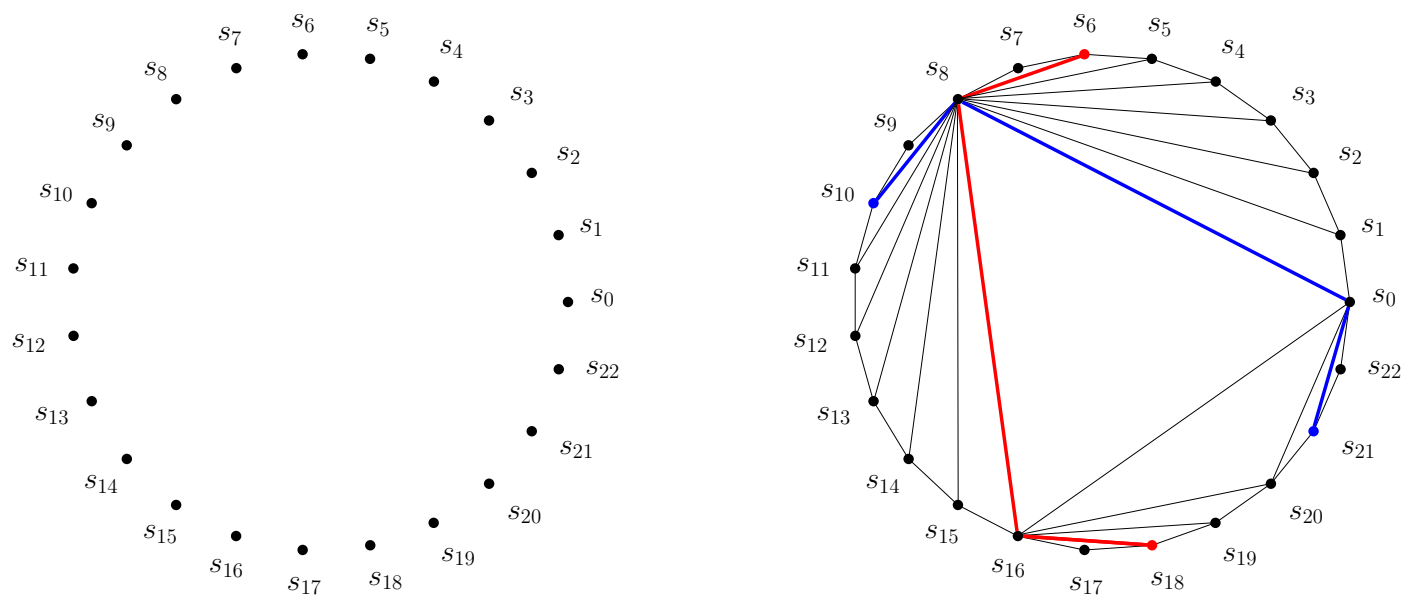

Figure 1: Left: The set $S$ of 23 points placed at the vertices of a regular 23-gon. Right: A triangulation of $S$ with stretch factor $\left(2 \sin \frac{2 \pi}{23}+\sin \frac{8 \pi}{23}\right) / \sin \frac{11 \pi}{23}=1.4308 \ldots$, which is achieved by the detours for the pairs $s_{10}, s_{21}$ and $s_{6}, s_{18}$. The shortest paths connecting the pairs are shown in blue and red, respectively.

Given a chord $s_{0} s_{i}$, let lower $\left(s_{0} s_{i}\right)=\left\{s_{i+1}, \ldots, s_{22}\right\}$ and upper $\left(s_{0} s_{i}\right)=\left\{s_{1}, \ldots s_{i-1}\right\}$. The range of possible convex hull lengths of the longest chord in a triangulation of $S$ is given by the following.

Lemma 1. If $\ell$ is the convex hull length of the longest chord in a triangulation of $S$, then $\ell \in$ $\{8,9,10,11\}$. 


\begin{tabular}{|c|c|c|c|c|}
\hline & $f(4,7)$ & $1.3396 \ldots$ & $\quad f(2,2,8)$ & $1.4308 \ldots$ \\
\hline & $f(5,6)$ & $1.3651 \ldots$ & $\quad f(3,3,5)$ & 1.4312 . \\
\hline 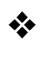 & $f(5,7)$ & $1.4514 \ldots$ & $f(3,4,4)$ & 1.4409 . \\
\hline$*$ & $f(6,6)$ & $1.4650 \ldots$ & $\quad f(1,4,7)$ & $1.4761 \ldots$ \\
\hline & $f(2,3,6)$ & $1.4023 \ldots$ & $f(2,3,7)$ & 1.4886 \\
\hline & $f(1,5,5)$ & $1.4061 \ldots$ & $\quad f(3,3,6)$ & $1.5312 \ldots$ \\
\hline$*$ & $f(2,4,5)$ & 1.4237 . & $\quad f(1,1,4,5)$ & 1.4263 . \\
\hline$*$ & $f(1,3,8)$ & $1.4257 \ldots$ & $f(1,2,3,5)$ & $1.4388 \ldots$ \\
\hline
\end{tabular}

Table 1: Relevant values of $f\left(n_{1}, \ldots, n_{k}\right)$ as required by the proofs in this section. Values used explicitly in the proofs are marked using $*$.

Proof. We clearly have $\ell \geq 2$. Since $S$ is symmetric, we can assume that $s_{0} s_{\ell}$ is the longest chord. Since $\mu(i, j) \leq 11$ for any $0 \leq i, j \leq 22$, we have $\ell \leq 11$. Suppose for contradiction that $2 \leq \ell \leq 7$. Then $s_{0} s_{\ell}$ is an edge of some triangle $\Delta s_{0} s_{\ell} s_{m}$, where $\ell+1 \leq m \leq 22$. In particular,

$$
\mu(0, \ell), \mu(\ell, m), \mu(0, m) \leq \ell \leq 7 \text {. }
$$

If $m \leq 11$, then $\mu(0, m)=\min (m, 23-m)=m \geq \ell+1$, a contradiction to $\ell$ 's maximality. Assume now that $m \geq 12$; then $\mu(0, m)=23-m \leq \ell$, since $\ell$ is the length of a longest chord. It follows that $m \geq 23-\ell \geq 23-7=16$. If $m-\ell \leq 11$, then $\mu(\ell, m)=m-\ell \geq 16-7=9$, a contradiction to (3). If $m-\ell \geq 12$, then $\mu(\ell, m)=23-(m-\ell)=23-m+\ell \geq \ell+1$, a contradiction to $\ell$ 's maximality. Consequently, we have $8 \leq \ell \leq 11$, as required.

Proof outline. For every $\ell \in\{8,9,10,11\}$, if the longest chord in a triangulation $T$ has length $\ell$, we show that $\delta(T) \geq f(2,4,5)=1.4237 \ldots$ Assuming that $s_{0} s_{\ell}$ is a longest chord, we consider the triangle with base $s_{0} s_{\ell}$ and third vertex in upper $\left(s_{0} s_{\ell}\right)$ or lower $\left(s_{0} s_{\ell}\right)$, depending on $\ell$. For each such triangle, we show that if the edges of the triangle along with the convex hull edges of $S$ are present, then in any resulting triangulation there is a pair whose stretch factor is at least $f(2,4,5)=1.4237 \ldots$ Essentially, the long chords act as obstacles which contribute to long detours for some point pairs. In four subsequent lemmas, we consider the convex hull lengths $8,9,10,11$ (from Lemma 1) successively.

In some arguments, we consider a primary pair $s_{i}, s_{j}$, and possible shortest paths between the two vertices. We show that if certain intermediate vertices are present in $\pi\left(s_{i}, s_{j}\right)$, then $\delta\left(s_{i}, s_{j}\right) \geq$ $f(2,4,5)$. Otherwise if certain long edges are present in $\pi\left(s_{i}, s_{j}\right)$, then $\delta\left(s_{u}, s_{v}\right) \geq f(2,4,5)$, where $s_{u}, s_{v}$ is a secondary pair. In the figures, wherever required, we use circles and squares to mark the primary and secondary pairs, respectively (see for instance Fig. 2). In some of the cases, a primary pair suffices in the argument, i.e., no secondary pair is needed.

Lemma 2. If $\ell=8$, then $\delta(T) \geq f(2,4,5)=1.4237 \ldots$

Proof. Refer to Fig. 2, Let $s_{0} s_{8}$ be the longest chord. The triangle with base $s_{0} s_{8}$ and third vertex in lower $\left(s_{0} s_{8}\right)$ has two other sides of convex hull lengths 7 and 8. It thus suffices to consider the triangle $\Delta s_{0} s_{8} s_{16}$ only and assume that the edges $s_{0} s_{8}, s_{8} s_{16}$ and $s_{0} s_{16}$ are present.

In this proof, the primary pair is $s_{10}, s_{21}$ and the secondary pair is $s_{3}, s_{14}$. Now, consider the pair $s_{10}, s_{21}$. Note that either $s_{0} \in \pi\left(s_{10}, s_{21}\right)$ or $s_{16} \in \pi\left(s_{10}, s_{21}\right)$. In the former case, $\delta\left(s_{10}, s_{21}\right) \geq$ $|\rho(10,8,0,21)| /\left|s_{10} s_{21}\right| \geq f(2,8,2)=1.4308 \ldots$ We may thus assume that $s_{16} \in \pi\left(s_{10}, s_{21}\right)$.

Similarly, for the pair $s_{3}, s_{14}$ either $s_{0} \in \pi\left(s_{3}, s_{14}\right)$ or $s_{8} \in \pi\left(s_{3}, s_{14}\right)$. If $s_{0} \in \pi\left(s_{3}, s_{14}\right)$, then $\delta\left(s_{3}, s_{14}\right) \geq|\rho(3,0,16,14)| /\left|s_{3} s_{14}\right| \geq f(3,7,2)=1.4886 \ldots$ Thus, assume that $s_{8} \in \pi\left(s_{3}, s_{14}\right)$. 

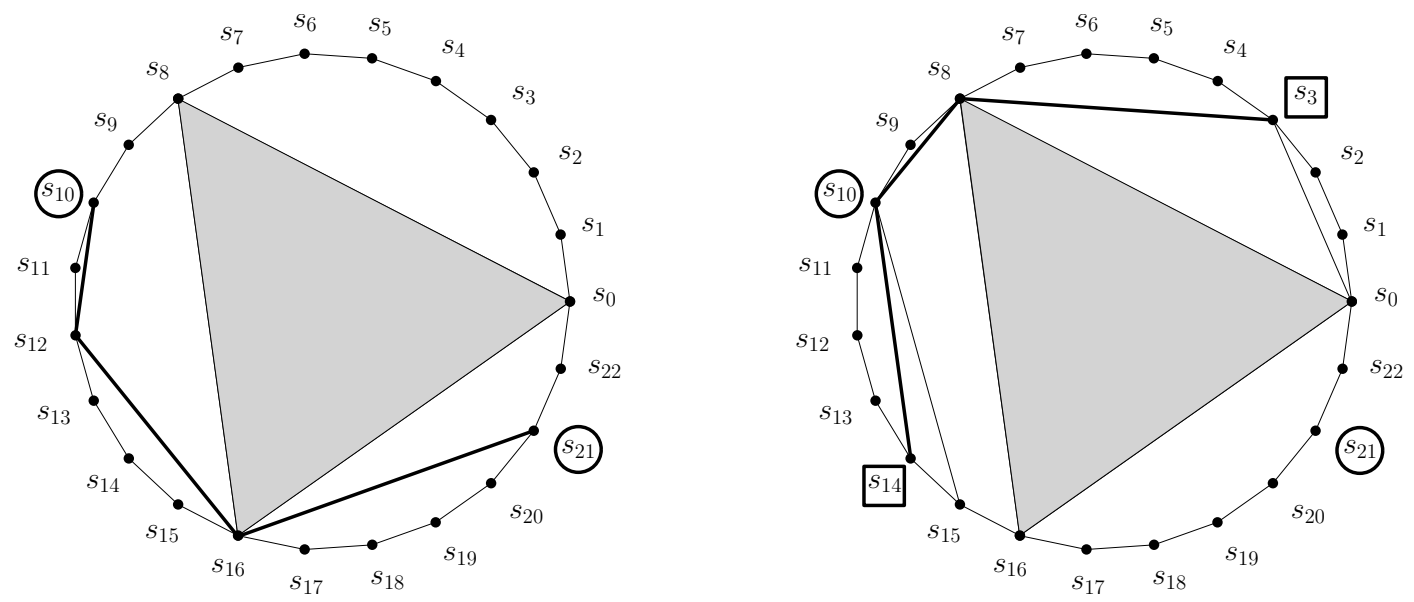

Figure 2: Illustrating Lemma 2 Left: $s_{12} \in \pi\left(s_{10}, s_{21}\right)$, primary pair: $s_{10}, s_{21}$. Right: $s_{10} s_{15} \in \pi\left(s_{10}, s_{21}\right)$, primary pair: $s_{10}, s_{21}$, secondary pair: $s_{3}, s_{14}$.

If at least one of $s_{12}, s_{13}$, or $s_{14}$ is in $\pi\left(s_{10}, s_{21}\right)$, then

$$
\begin{aligned}
\delta\left(s_{10}, s_{21}\right) & \geq \frac{\min (|\rho(10,12,16,21)|,|\rho(10,13,16,21)|,|\rho(10,14,16,21)|)}{\left|s_{10} s_{21}\right|} \\
& \geq \min (f(2,4,5), f(3,3,5), f(4,2,5))=f(2,4,5)=1.4237 \ldots
\end{aligned}
$$

Otherwise, one of $s_{10} s_{15}, s_{10} s_{16}, s_{11} s_{15}$, or $s_{11} s_{16}$ must be in $\pi\left(s_{10}, s_{21}\right)$, and

$$
\begin{aligned}
\delta\left(s_{3}, s_{14}\right) & \geq \frac{\min (|\rho(3,8,10,14)|,|\rho(3,8,11,14)|),|\rho(3,8,15,14)|)}{\left|s_{3} s_{14}\right|} \\
& \geq \min (f(5,2,4), f(5,3,3), f(1,5,7))=f(2,4,5)=1.4237 \ldots
\end{aligned}
$$

Lemma 3. If $\ell=9$, then $\delta(T) \geq f(2,4,5)=1.4237 \ldots$

Proof. Let $s_{0} s_{9}$ be the longest chord and consider the triangle with base $s_{0} s_{9}$ and the third vertex in lower $\left(s_{0} s_{9}\right)$. There are three possible cases depending on the convex hull lengths of other two sides of the triangle: $\{7,7\},\{8,6\}$ or $\{9,5\}$. We consider them successively.
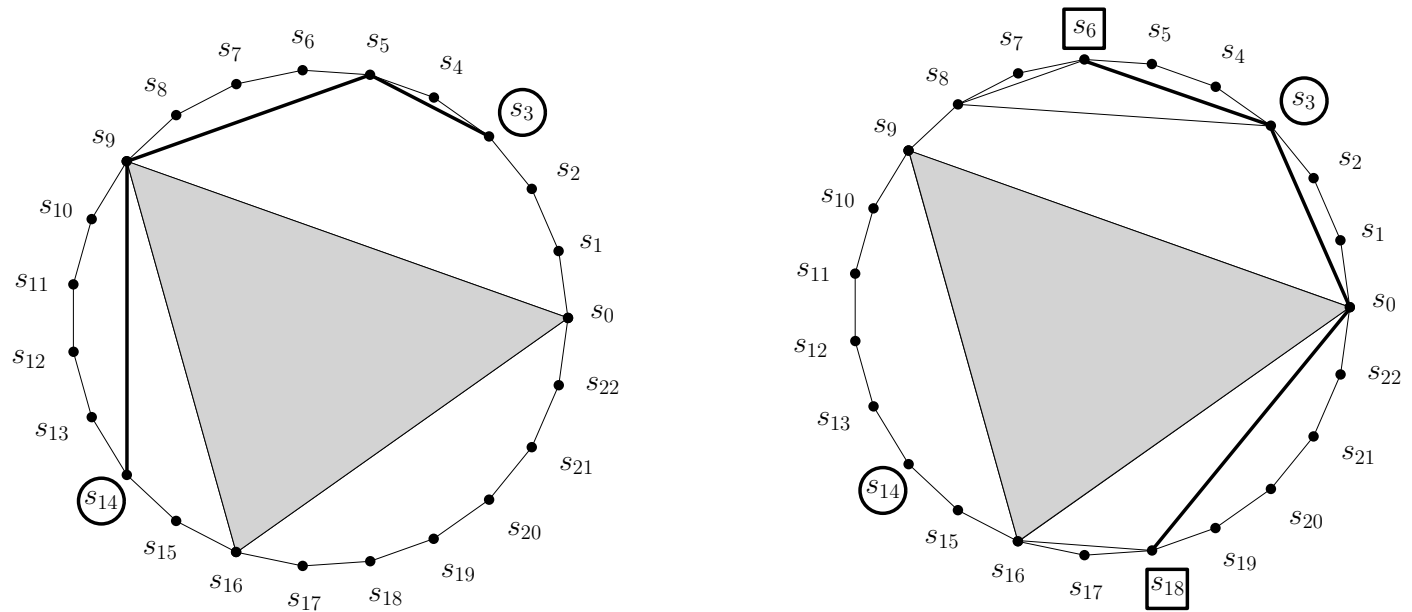

Figure 3: Illustrating CASE A from Lemma 3 Left: $s_{5} \in \pi\left(s_{3}, s_{14}\right)$. Right: $s_{3} s_{8} \in \pi\left(s_{3}, s_{14}\right)$. 
CAse A: The convex hull lengths of the other two sides are $\{7,7\}$. Let $\Delta s_{0} s_{9} s_{16}$ be the required triangle; refer to Fig. 3. In this case, the primary pair is $s_{3}, s_{14}$ and the secondary pair is $s_{6}, s_{18}$. Either $s_{0} \in \pi\left(s_{3}, s_{14}\right)$ or $s_{9} \in \pi\left(s_{3}, s_{14}\right)$. If $s_{0} \in \pi\left(s_{3}, s_{14}\right)$, then $\delta\left(s_{3}, s_{14}\right) \geq|\rho(3,0,16,14)| /\left|s_{3} s_{14}\right| \geq$ $f(3,7,2)=1.4886 \ldots$ Thus, we assume that $s_{9} \in \pi\left(s_{3}, s_{14}\right)$.

Similarly, for the pair $s_{6}, s_{18}$, either $s_{9} \in \pi\left(s_{6}, s_{18}\right)$ or $s_{0} \in \pi\left(s_{6}, s_{18}\right)$. If $s_{9} \in \pi\left(s_{6}, s_{18}\right)$ then $\delta\left(s_{6}, s_{18}\right) \geq|\rho(6,9,16,18)| /\left|s_{6} s_{18}\right| \geq f(3,7,2)=1.4886 \ldots$ Thus, assume that $s_{0} \in \pi\left(s_{6}, s_{18}\right)$.

Now, if at least one of $s_{5}, s_{6}$, or $s_{7}$ is in $\pi\left(s_{3}, s_{14}\right)$, then

$$
\begin{aligned}
\delta\left(s_{3}, s_{14}\right) & \geq \frac{\min (|\rho(3,5,9,14)|,|\rho(3,6,9,14)|,|\rho(3,7,9,14)|)}{\left|s_{3} s_{14}\right|} \\
& \geq \min (f(2,4,5), f(3,3,5), f(4,2,5))=f(2,4,5)=1.4237 \ldots
\end{aligned}
$$

Otherwise, one of $s_{3} s_{8}, s_{3} s_{9}, s_{4} s_{8}$, or $s_{4} s_{9}$ must be in $\pi\left(s_{3}, s_{14}\right)$, and

$$
\delta\left(s_{6}, s_{18}\right) \geq \frac{\min (|\rho(6,3,0,18)|,|\rho(6,4,0,18)|)}{\left|s_{6} s_{18}\right|} \geq \min (f(3,3,5), f(2,4,5))=f(2,4,5)=1.4237 \ldots
$$

CASE B: The convex hull lengths of the other two sides are $\{8,6\}$. Let $\Delta s_{0} s_{9} s_{17}$ be the required triangle; refer to Fig. 4 (left). As in CASE A, the primary pair is $s_{3}, s_{14}$ and the secondary pair is $s_{6}, s_{18}$. Consider the pair $s_{3}, s_{14}$. Either $s_{17} \in \pi\left(s_{3}, s_{14}\right)$ or $s_{9} \in \pi\left(s_{3}, s_{14}\right)$. If $s_{17} \in \pi\left(s_{3}, s_{14}\right)$, then $\delta\left(s_{3}, s_{14}\right) \geq|\rho(3,0,17,14)| /\left|s_{3} s_{14}\right| \geq f(3,6,3)=1.5312 \ldots$ So we assume that $s_{9} \in \pi\left(s_{3}, s_{14}\right)$.
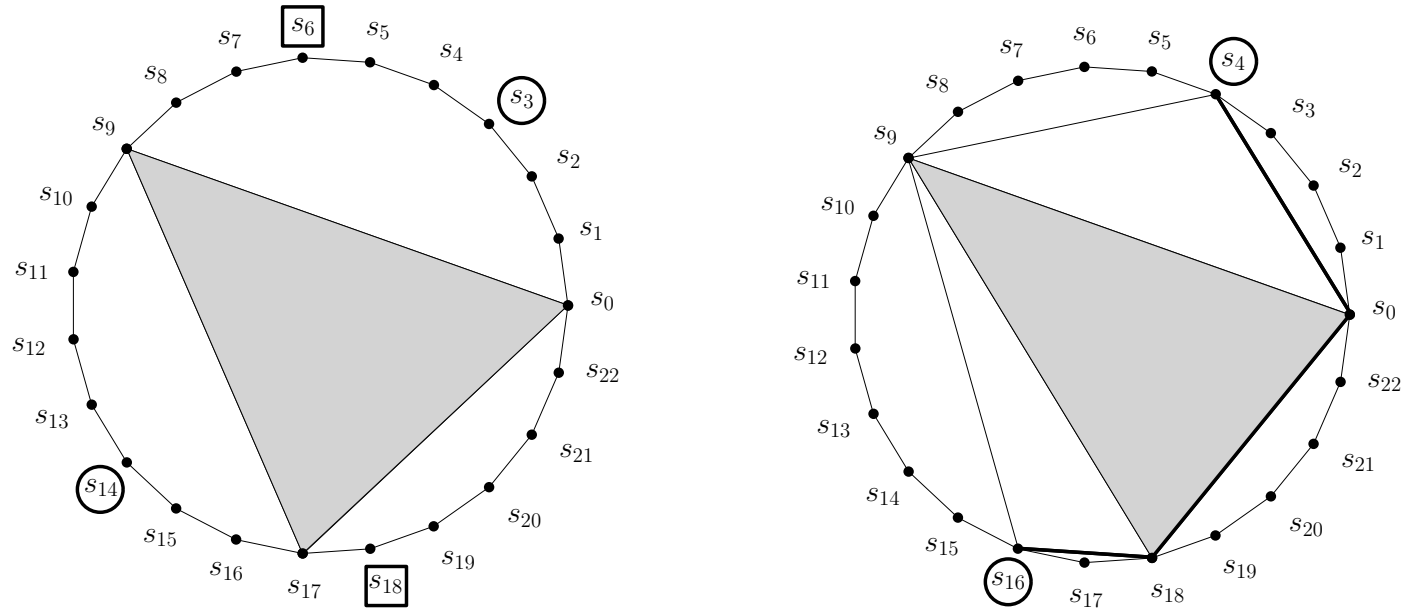

Figure 4: Illustrating CASE B (left) and CASE C (right) from Lemma 3

Similarly, for the pair $s_{6}, s_{18}$, either $s_{9} \in \pi\left(s_{6}, s_{18}\right)$ or $s_{0} \in \pi\left(s_{6}, s_{18}\right)$. If $s_{9} \in \pi\left(s_{6}, s_{18}\right)$ then $\delta\left(s_{6}, s_{18}\right) \geq|\rho(6,9,17,18)| /\left|s_{6} s_{18}\right| \geq f(3,8,1)=1.4257 \ldots$ Thus, we assume that $s_{0} \in \pi\left(s_{6}, s_{18}\right)$. Now, it can be checked that by the same analysis as in CASE A, the same lower bound of $f(2,4,5)$ holds.

CASE C: The convex hull lengths of the other two sides of the triangle are $\{9,5\}$. Let $\Delta s_{0} s_{9} s_{18}$ be the required triangle; refer to Fig. 4 (right). Then,

$$
\delta\left(s_{4}, s_{16}\right) \geq \frac{\min (|\rho(4,0,18,16)|,|\rho(4,9,16)|)}{\left|s_{4} s_{16}\right|} \geq \min (f(4,5,2), f(5,7))=f(2,4,5)=1.4237 \ldots
$$

Lemma 4. If $\ell=10$, then $\delta(T) \geq f(2,4,5)=1.4237 \ldots$ 

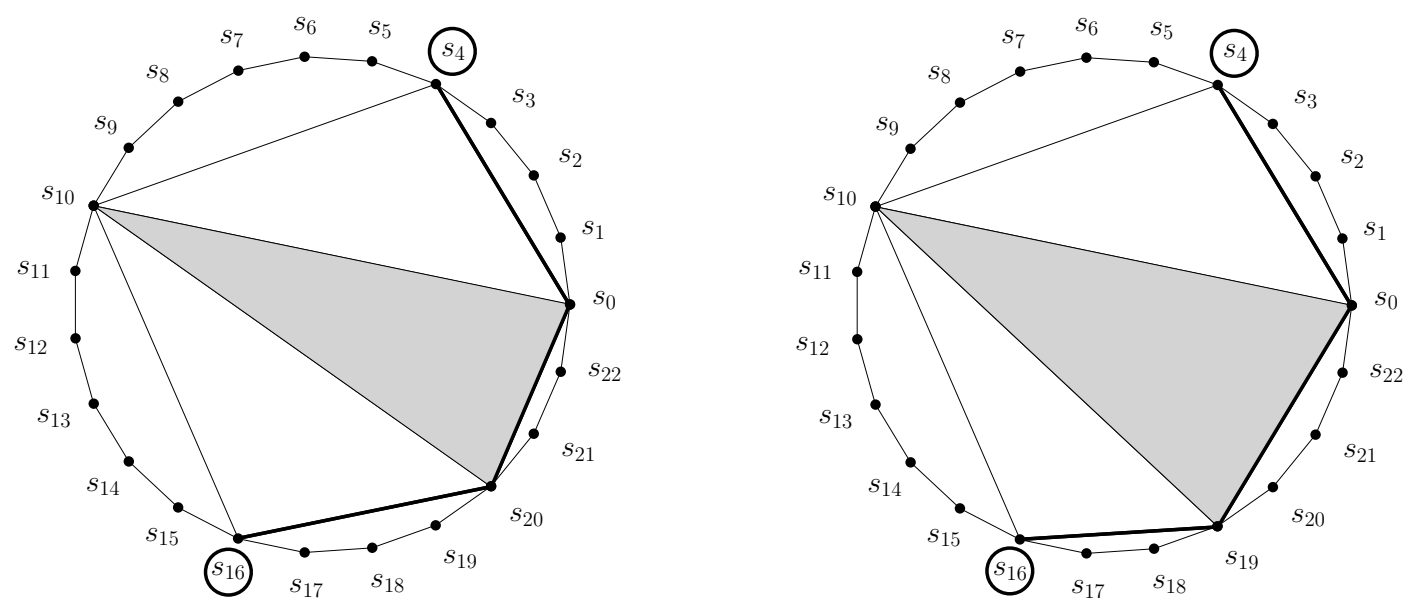

Figure 5: Illustrating CASE A from Lemma 4

Proof. Let $s_{0} s_{10}$ be the longest chord. The possible convex hull lengths of the other two sides of the triangle with base $s_{0} s_{10}$ and the third vertex in lower $\left(s_{0} s_{10}\right)$ are $\{10,3\},\{9,4\},\{8,5\},\{7,6\}$. We consider these cases successively.

CAsE A: The convex hull lengths of the other two sides of the triangle are $\{10,3\},\{9,4\}$ or $\{8,5\}$. Let $\Delta s_{0} s_{10} s_{20}, \Delta s_{0} s_{10} s_{19}, \Delta s_{0} s_{10} s_{18}$ be the required triangles, respectively; refer to Fig. 5 and Fig. 6(left). Then,

$$
\begin{aligned}
\delta\left(s_{4}, s_{16}\right) & \geq \frac{\min (|\rho(4,0,20,16)|,|\rho(4,0,19,16)|,|\rho(4,0,18,16)|,|\rho(4,10,16)|)}{\left|s_{4} s_{16}\right|} \\
& \geq \min (f(4,3,4), f(4,4,3), f(4,5,2), f(6,6))=f(2,4,5)=1.4237 \ldots
\end{aligned}
$$
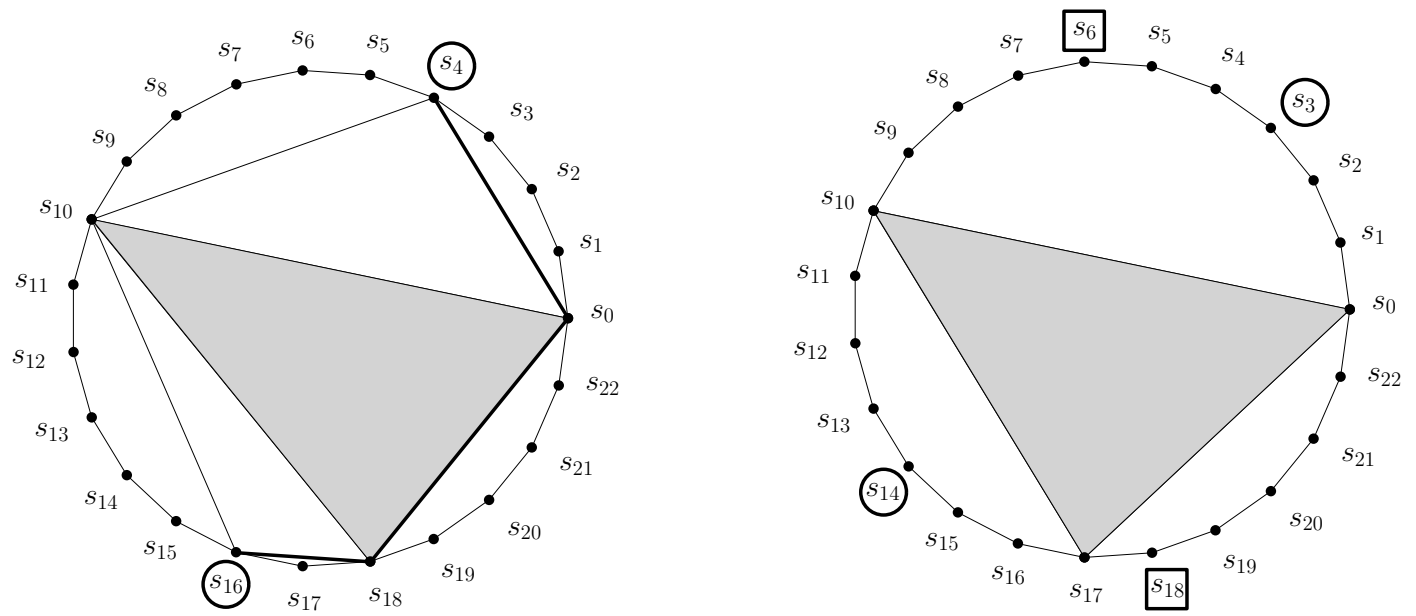

Figure 6: Illustrating CASE A (left) and CASE B (right) from Lemma 4

CASE B: The convex hull lengths of the other two sides are $\{7,6\}$. Let $\Delta s_{0} s_{10} s_{17}$ be the required triangle; refer to Fig. 6(right). In this case, the primary pair is $s_{3}, s_{14}$ and the secondary pair is $s_{6}, s_{18}$. Either $s_{0} \in \pi\left(s_{3}, s_{14}\right)$ or $s_{10} \in \pi\left(s_{3}, s_{14}\right)$. If $s_{0} \in \pi\left(s_{3}, s_{14}\right)$, then $\delta\left(s_{3}, s_{14}\right) \geq$ $|\rho(3,0,17,14)| /\left|s_{3} s_{14}\right| \geq f(3,6,3)=1.5312 \ldots$ Thus, we assume that $s_{10} \in \pi\left(s_{3}, s_{14}\right)$.

Similarly, for the pair $s_{6}, s_{18}$, either $s_{10} \in \pi\left(s_{6}, s_{18}\right)$ or $s_{0} \in \pi\left(s_{6}, s_{18}\right)$. If $s_{10} \in \pi\left(s_{6}, s_{18}\right)$, then $\delta\left(s_{6}, s_{18}\right) \geq|\rho(6,10,17,18)| /\left|s_{6} s_{18}\right| \geq f(4,7,1)=1.4761 \ldots$ Thus, assume that $s_{0} \in \pi\left(s_{6}, s_{18}\right)$. 
Now, if at least one of $s_{5}, s_{6}, s_{7}$, or $s_{8}$ is in $\pi\left(s_{3}, s_{14}\right)$, then

$$
\begin{aligned}
\delta\left(s_{3}, s_{14}\right) & \geq \frac{\min (|\rho(3,5,10,14)|,|\rho(3,6,10,14)|,|\rho(3,7,10,14)|,|\rho(3,8,10,14)|)}{\left|s_{3} s_{14}\right|} \\
& \geq \min (f(2,5,4), f(3,4,4), f(4,3,4), f(5,2,4))=f(2,4,5)=1.4237 \ldots
\end{aligned}
$$

Otherwise, one of $s_{3} s_{9}, s_{3} s_{10}, s_{4} s_{9}$, or $s_{4} s_{10}$ must be in $\pi\left(s_{3}, s_{14}\right)$, and

$$
\begin{aligned}
\delta\left(s_{6}, s_{18}\right) & \geq \frac{\min (|\rho(6,3,0,18)|,|\rho(6,4,0,18)|)}{\left|s_{6} s_{18}\right|} \\
& \geq \min (f(3,3,5), f(2,4,5))=f(2,4,5)=1.4237 \ldots
\end{aligned}
$$

Lemma 5. If $\ell=11$, then $\delta(T) \geq f(2,4,5)=1.4237 \ldots$

Proof. Let $s_{0} s_{11}$ be the longest chord. Since the size of upper $\left(s_{0} s_{11}\right)$ is smaller than the size of lower $\left(s_{0} s_{11}\right)$, we consider upper $\left(s_{0} s_{11}\right)$ is our analysis. The possible convex hull lengths of the other two sides of the triangle with base $s_{0} s_{11}$ and the third vertex in upper $\left(s_{0} s_{11}\right)$ are $\{1,10\}$, $\{2,9\},\{3,8\},\{4,7\},\{5,6\}$. We consider the following cases successively.
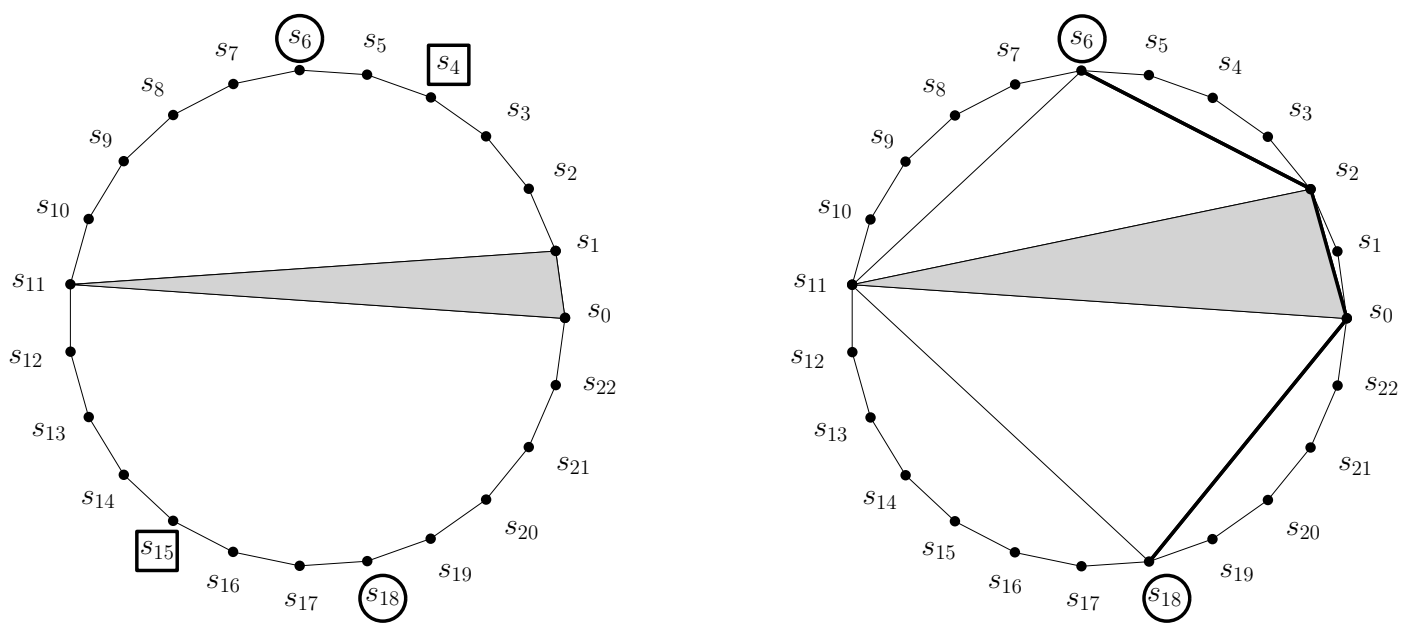

Figure 7: Illustrating CASE A (left) and CASE B (right) from Lemma 5 .

CASE A: The convex hull lengths of the other two sides are $\{1,10\}$. Let $\Delta s_{0} s_{1} s_{11}$ be the required triangle; refer to Fig. 7 (left). In this case, the primary pair is $s_{6}, s_{18}$ and the secondary pair is $s_{4}, s_{15}$. Consider the pair $s_{6}, s_{18}$. Either $s_{11} \in \pi\left(s_{6}, s_{18}\right)$ or $s_{0} \in \pi\left(s_{6}, s_{18}\right)$. If $s_{11} \in \pi\left(s_{6}, s_{18}\right)$, then $\delta\left(s_{6}, s_{18}\right) \geq|\rho(6,11,18)| /\left|s_{6} s_{18}\right| \geq f(5,7)=1.4514 \ldots$ Hence, we assume that $s_{0} \in \pi\left(s_{6}, s_{18}\right)$.

If at least one of $s_{2}, s_{3}, s_{4}$, or $s_{5}$ is in $\pi\left(s_{6}, s_{18}\right)$, then

$$
\begin{aligned}
\delta\left(s_{6}, s_{18}\right) & \geq \frac{\min (|\rho(6,2,1,0,18)|,|\rho(6,3,1,0,18)|,|\rho(6,4,1,0,18)|,|\rho(6,5,1,0,18)|)}{\left|s_{6} s_{18}\right|} \\
& =\min (f(4,1,1,5), f(3,2,1,5), f(2,3,1,5), f(1,4,1,5))=f(1,1,4,5)=1.4263 \ldots
\end{aligned}
$$

Otherwise, $s_{1} s_{6}$ is in $\pi\left(s_{6}, s_{18}\right)$, and then

$$
\delta\left(s_{4}, s_{15}\right) \geq \frac{\min (|\rho(4,6,11,15)|,|\rho(4,1,0,15)|)}{\left|s_{4} s_{15}\right|} \geq \min (f(2,5,4), f(3,1,8))=f(2,4,5)=1.4237 \ldots
$$



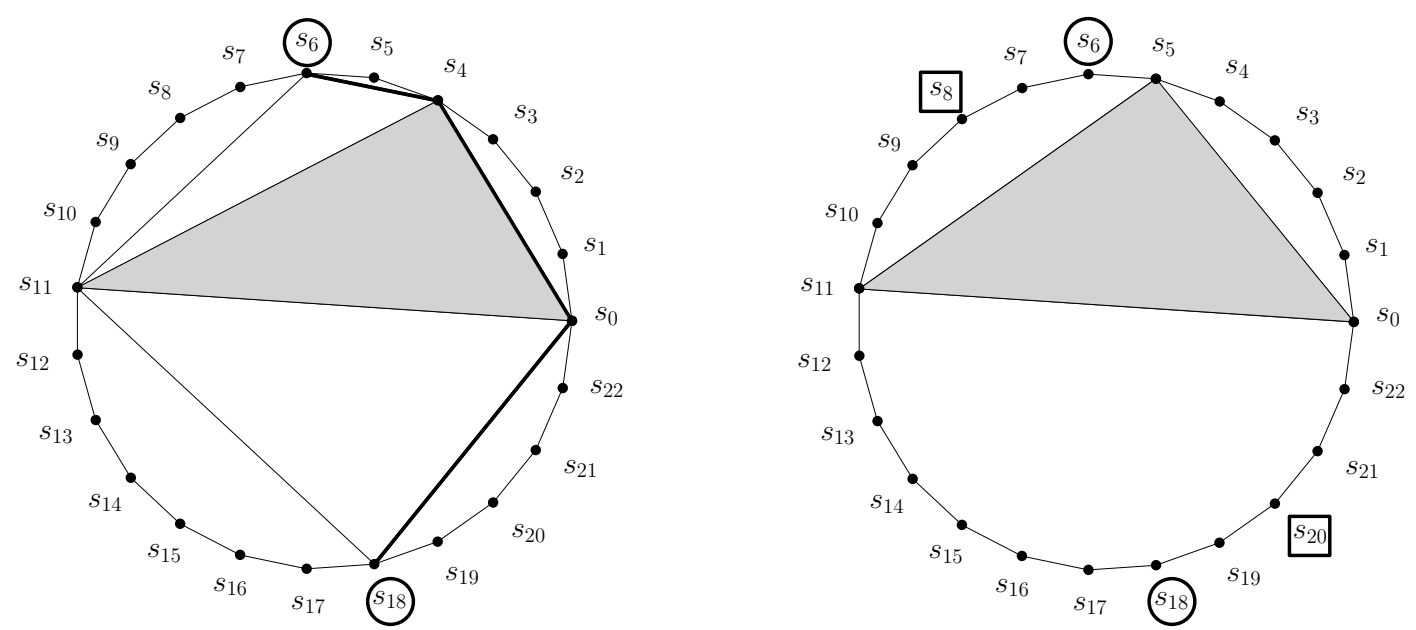

Figure 8: Illustrating CASE B (left) and CASE C (right) from Lemma 5

CASE B: The convex hull lengths of the other two sides are $\{2,9\},\{3,8\}$ or $\{4,7\}$. Let $\Delta s_{0} s_{2} s_{11}$, $\Delta s_{0} s_{3} s_{11}, \Delta s_{0} s_{4} s_{11}$ be the required triangles, respectively. Refer to Fig. 7 (right) and Fig. 8 (left) for illustrations.

As in the CASE $\mathrm{A}$, we may assume that $s_{0} \in \pi\left(s_{6}, s_{18}\right)$. Then,

$$
\begin{aligned}
\delta\left(s_{6}, s_{18}\right) & \geq \frac{\min (|\rho(6,2,0,18)|,|\rho(6,3,0,18)|,|\rho(6,4,0,18)|)}{\left|s_{6} s_{18}\right|} \\
& \geq \min (f(4,2,5), f(3,3,5), f(2,4,5))=f(2,4,5)=1.4237 \ldots
\end{aligned}
$$

CASE C: The convex hull lengths of the other two sides are $\{5,6\}$. Let $\Delta s_{0} s_{5} s_{11}$ be the required triangle; refer to Fig. 8 (right). In this case, the primary pair is $s_{6}, s_{18}$ and the secondary pair is $s_{8}, s_{20}$. As in the CASE A, we assume that $s_{0} \in \pi\left(s_{6}, s_{18}\right)$.

Now, if at least one of $s_{19}, s_{20}, s_{21}$, or $s_{22}$ is in $\pi\left(s_{6}, s_{18}\right)$, then

$$
\begin{aligned}
\delta\left(s_{6}, s_{18}\right) & \geq \frac{\min (|\rho(6,5,0,19,18)|,|\rho(6,5,0,20,18)|,|\rho(6,5,0,21,18)|,|\rho(6,5,0,22,18)|)}{\left|s_{6} s_{18}\right|} \\
& =\min (f(1,5,4,1), f(1,5,3,2), f(1,5,2,3), f(1,5,1,4))=f(1,1,4,5)=1.4263 \ldots
\end{aligned}
$$

Otherwise, $s_{0} s_{18}$ is in $\pi\left(s_{6}, s_{18}\right)$, and then

$$
\begin{aligned}
\delta\left(s_{8}, s_{20}\right) & \geq \frac{\min (|\rho(8,5,0,20)|,|\rho(8,11,18,20)|)}{\left|s_{8} s_{20}\right|} \\
& \geq \min (f(3,5,3), f(3,7,2))=f(3,3,5)=1.4312 \ldots
\end{aligned}
$$

Putting these facts together yields the main result of this section:

Theorem 1. Let $S$ be a set of 23 points placed at the vertices of a regular 23-gon. Then

$$
\delta_{0}(S)=f(2,2,8)=\left(2 \sin \frac{2 \pi}{23}+\sin \frac{8 \pi}{23}\right) / \sin \frac{11 \pi}{23}=1.4308 \ldots
$$

Proof. By Lemmas 2,5, we conclude that $\delta_{0}(S) \geq f(2,4,5)=\left(\sin \frac{2 \pi}{23}+\sin \frac{4 \pi}{23}+\sin \frac{5 \pi}{23}\right) / \sin \frac{11 \pi}{23}=$ $1.4237 \ldots$ On the other hand, the triangulation of $S$ in Fig. 1 (right) has stretch factor $f(2,2,8)=$ $1.4308 \ldots$ and thus $f(2,4,5)=1.4237 \ldots \leq \delta_{0}(S) \leq f(2,2,8)=1.4308 \ldots$ 
A parallel C++ program 2 that generates all triangulations of $S$ based on a low memory algorithm by Parvez et al. [36, Section 4] shows that each of the $C_{21}$ triangulations has stretch factor at least $f(2,2,8)$. We thereby obtain the following final result: $\delta_{0}(S)=f(2,2,8)=\left(2 \sin \frac{2 \pi}{23}+\right.$ $\left.\sin \frac{8 \pi}{23}\right) / \sin \frac{11 \pi}{23}=1.4308 \ldots$

Remarks. Using the program we have also checked that the next largest stretch factor among all triangulations is $f(3,3,5)=1.4312 \ldots$, and further that there is no triangulation of $S$ that has stretch-factor $<1.4312$ other than $f(2,2,8)$. Thus, the result in Theorem 1 is not affected by floating-point precision errors.
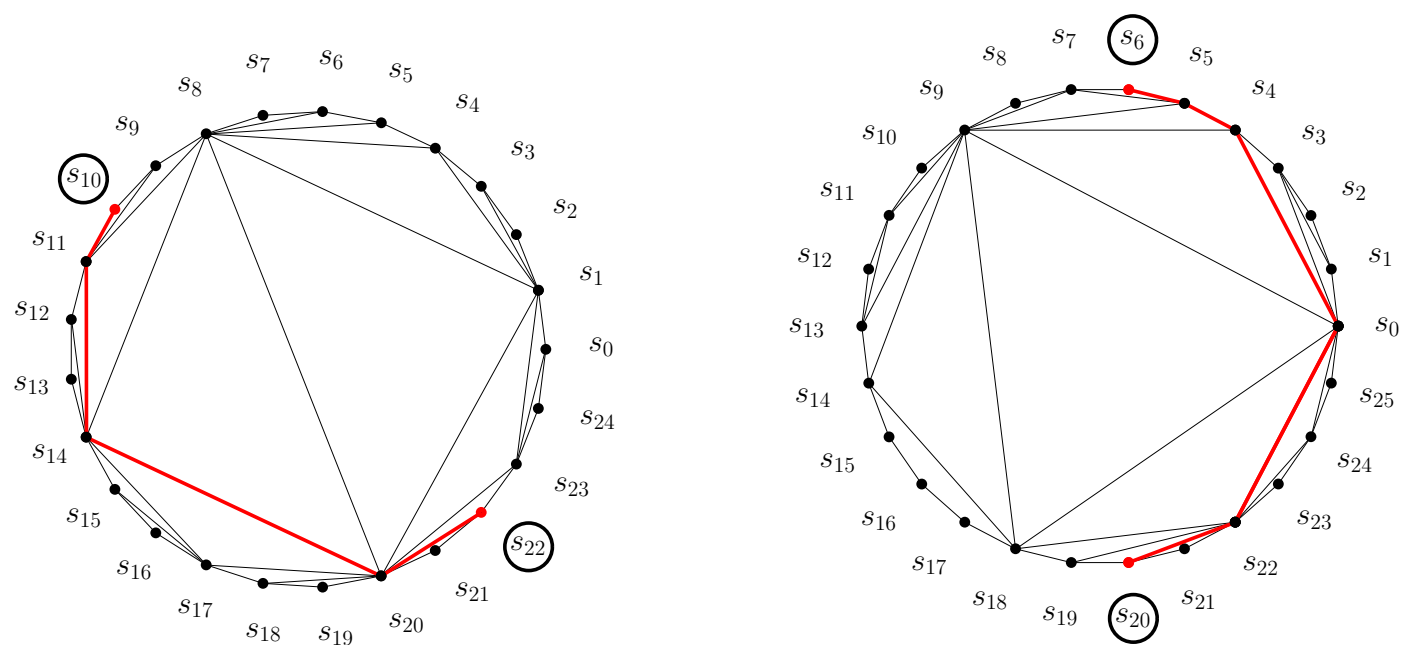

Figure 9: Triangulations of $S_{25}$ and $S_{26}$ with stretch factors $<1.4296$ and $<1.4202$, respectively. Worst stretch factor pairs are marked in circles and the corresponding shortest paths are shown in red.

Let $S_{n}$ denote the set of points placed at the vertices of a regular $n$-gon. Using a computer program, Mulzer obtained the values $\delta_{0}\left(S_{n}\right)$ for $4 \leq n \leq 21$ in his thesis [34, Chapter 3]. Using our $\mathrm{C}++$ program, we confirmed the previous values and extended the range up to $n=24: \delta_{0}\left(S_{22}\right)=$ $1.4047 \ldots, \delta_{0}\left(S_{24}\right)=1.4013 \ldots$ and somewhat surprisingly, $\delta_{0}\left(S_{23}\right)=1.4308 \ldots$ By upper bound constructions, it follows that $\delta_{0}\left(S_{25}\right)<1.4296$ and $\delta_{0}\left(S_{26}\right)<1.4202$; see Fig. 9. Observe that $\delta_{0}\left(S_{n}\right)$ does not exhibit a monotonic behavior; see Table 2

\begin{tabular}{||c|c||c|c||c|c||}
\hline$n$ & $\delta_{0}\left(S_{n}\right)$ & $n$ & $\delta_{0}\left(S_{n}\right)$ & $n$ & $\delta_{0}\left(S_{n}\right)$ \\
\hline 4 & $1.4142 \ldots$ & 12 & $1.3836 \ldots$ & 20 & $1.4142 \ldots$ \\
5 & $1.2360 \ldots$ & 13 & $1.3912 \ldots$ & 21 & $1.4161 \ldots$ \\
6 & $1.3660 \ldots$ & 14 & $1.4053 \ldots$ & 22 & $1.4047 \ldots$ \\
7 & $1.3351 \ldots$ & 15 & $1.4089 \ldots$ & $\mathbf{2 3}$ & $\mathbf{1 . 4 3 0 8 \ldots}$ \\
8 & $1.4142 \ldots$ & 16 & $1.4092 \ldots$ & 24 & $1.4013 \ldots$ \\
9 & $1.3472 \ldots$ & 17 & $1.4084 \ldots$ & 25 & $<1.4296$ \\
10 & $1.3968 \ldots$ & 18 & $1.3816 \ldots$ & 26 & $<1.4202$ \\
11 & $1.3770 \ldots$ & 19 & $1.4098 \ldots$ & & \\
\hline
\end{tabular}

Table 2: The values of $\delta_{0}\left(S_{n}\right)$ for $n=4, \ldots, 26$.

\footnotetext{
${ }^{2}$ Refer to the .cpp file and the Appendix within the source at arXiv:1509.07181.
} 


\section{Lower bounds for the degree 3 and 4 dilation}

In this section, we provide lower bounds for the worst case degree 3 and 4 dilation of point sets in the Euclidean plane. We begin with degree 3 dilation. We first present a set $P$ of $n=13$ points (a section of the hexagonal lattice with six boundary points removed) that has $\delta_{0}(P, 3) \geq 1+\sqrt{3}$ and then extend $P$ to achieve this lower bound for any $n>13$.

Theorem 2. For every $n \geq 13$, there exists a set $S$ of $n$ points such that $\delta_{0}(S, 3) \geq 1+\sqrt{3}=$ $2.7321 \ldots$ The inequality is tight for the presented sets.

Proof. Let $P=\left\{p_{0}\right\} \cup P_{1} \cup P_{2}$ be a set of 13 points as shown in Fig. 10 (left) where $P_{1}=$ $\left\{p_{1}, p_{3}, p_{5}, p_{7}, p_{9}, p_{11}\right\}$ and $P_{2}=\left\{p_{2}, p_{4}, p_{6}, p_{8}, p_{10}, p_{12}\right\}$. The points in $P_{1}$ and $P_{2}$ lie on the vertices
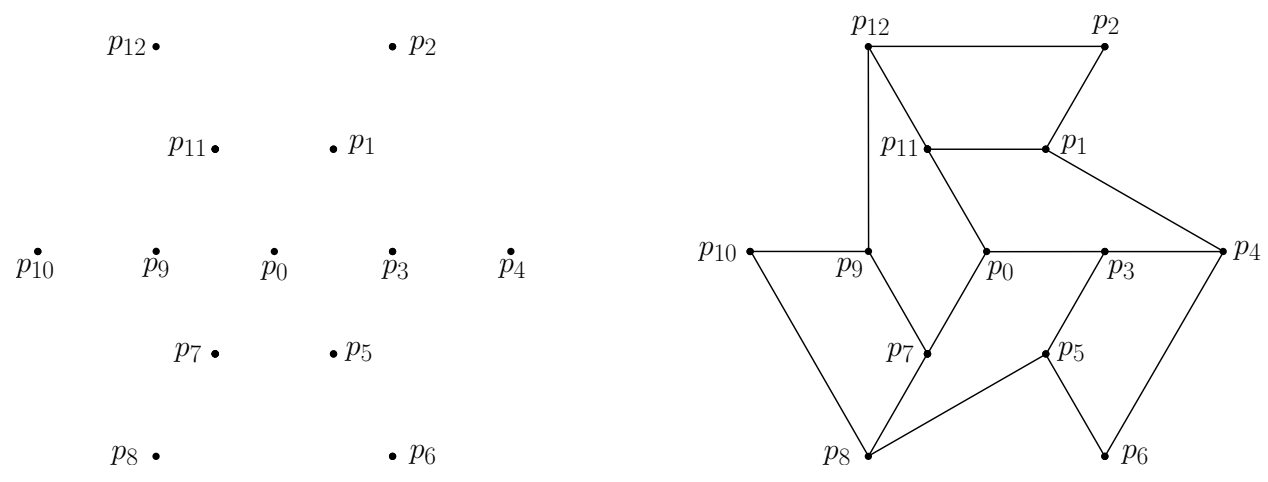

Figure 10: Left: the point set $P=\left\{p_{0}, p_{1}, \ldots, p_{12}\right\}$; some pairwise distances are: $\left|p_{2} p_{12}\right|=2,\left|p_{2} p_{3}\right|=$ $\left|p_{1} p_{5}\right|=\left|p_{1} p_{12}\right|=\sqrt{3}$. Right: a plane degree 3 geometric spanner on $P$ with stretch factor $1+\sqrt{3}$, which is achieved by the detours for the point pairs $\left\{p_{1}, p_{3}\right\},\left\{p_{5}, p_{7}\right\}$ and $\left\{p_{9}, p_{11}\right\}$.

of two regular homothetic hexagons centered at $p_{0}$ of radius 1 and 2 respectively. Furthermore, the points in each of the sets $\left\{p_{2}, p_{1}, p_{0}, p_{7}, p_{8}\right\},\left\{p_{4}, p_{3}, p_{0}, p_{9}, p_{10}\right\}$ and $\left\{p_{12}, p_{11}, p_{0}, p_{5}, p_{6}\right\}$ are collinear.

We show that $\delta_{0}(P, 3) \geq 1+\sqrt{3}$. Since no edge can contain a point in its interior, the point $p_{0}$ can have connecting edges only with the points from $P_{1}$. First, assume that the six edges in $E=\left\{p_{1} p_{3}, p_{3} p_{5}, p_{5} p_{7}, p_{7} p_{9}, p_{9} p_{11}, p_{1} p_{11}\right\}$ are present (see Fig. 11 (left)).

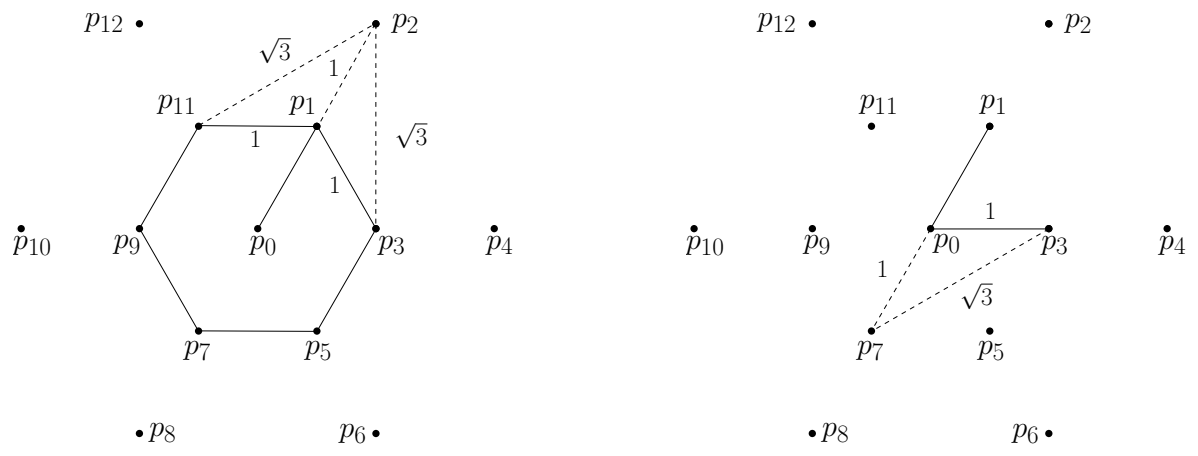

Figure 11: Left: all edges in $E$ are present. Right: CASE B.

We can also assume that the edge $p_{0} p_{1}$ is present since $p_{0}$ must be connected to at least one of the points in $P_{1}$. Observe that now $\operatorname{deg}\left(p_{1}\right)=3$. In this case,

$$
\delta\left(p_{1}, p_{2}\right) \geq \frac{|\rho(1, i, 2)|}{\left|p_{1} p_{2}\right|} \geq 1+\sqrt{3}, \text { where } i \in\{3,11\}
$$


Now assume that an edge in $E$, say $p_{1} p_{3}$, is missing. Then, the following three cases arise depending on $\operatorname{deg}\left(p_{0}\right) \in\{1,2,3\}$.

CASE A: If $\operatorname{deg}\left(p_{0}\right)=1$, then

$$
\delta\left(p_{1}, p_{3}\right) \geq \frac{|\rho(1, i, 3)|}{\left|p_{1} p_{3}\right|} \geq 1+\sqrt{3} \text { where } i \in\{2,5,4,11\} .
$$

CASE B: If $\operatorname{deg}\left(p_{0}\right)=2$, consider the edges $p_{0} p_{1}, p_{0} p_{3}$; see Fig. 11(right). If $p_{0} p_{1}, p_{0} p_{3}$ are present $\delta\left(p_{0}, p_{7}\right) \geq|\rho(0,3,7)| /\left|p_{0} p_{7}\right|=1+\sqrt{3}$ else if at least one edge in $\left\{p_{0} p_{1}, p_{0} p_{3}\right\}$ is absent then since $p_{1} p_{3}$ is absent, $\delta\left(p_{1}, p_{3}\right) \geq 1+\sqrt{3}$ by the same analysis as in CASE A.

CASE C: If $\operatorname{deg}\left(p_{0}\right)=3$, then if at least one of the edges $p_{0} p_{1}, p_{0} p_{3}$ is absent, $\delta\left(p_{1}, p_{3}\right) \geq 1+\sqrt{3}$ as shown in CASE A. Thus, assume that $p_{0} p_{1}, p_{0} p_{3}$ are present. Now, the following two non-symmetric cases will arise. Either $p_{0} p_{5}$ is present or $p_{0} p_{7}$ is present.

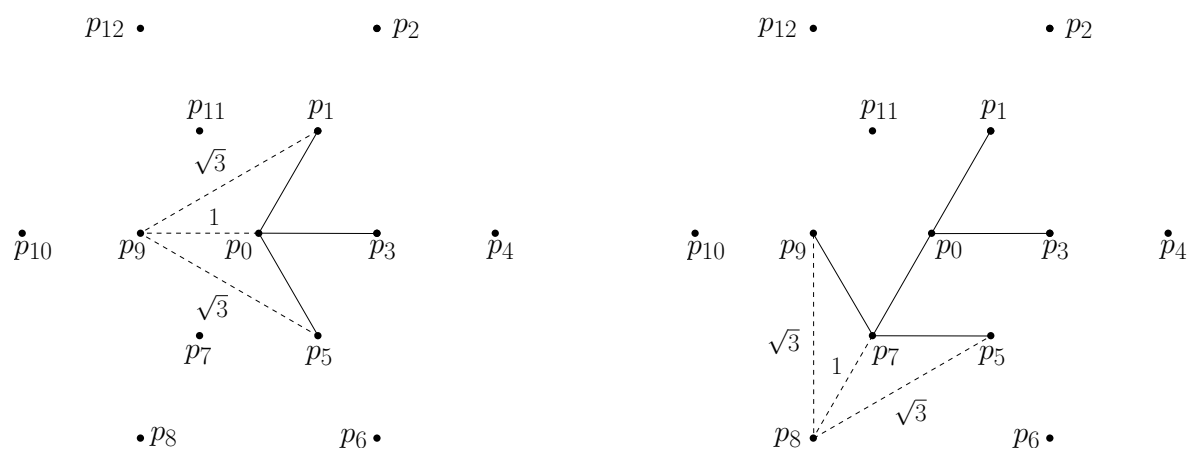

Figure 12: Left: the edges $p_{0} p_{1}, p_{0} p_{3}, p_{0} p_{5}$ are present. Right: the edges $p_{0} p_{1}, p_{0} p_{3}, p_{0} p_{7}$ are present.

If $p_{0} p_{5}$ is present (refer to Fig. 12 (left)) then,

$$
\delta\left(p_{0}, p_{9}\right) \geq \frac{|\rho(0, i, 9)|}{\left|p_{0} p_{9}\right|} \geq 1+\sqrt{3}, \text { where } i \in\{1,5\} .
$$

Now assume that $p_{0} p_{7}$ is present (refer to Fig. 12 (right)). Observe that if $p_{7} p_{9}$ is absent then,

$$
\delta\left(p_{7}, p_{9}\right) \geq \frac{|\rho(7, i, 9)|}{\left|p_{7} p_{9}\right|} \geq 1+\sqrt{3}, \text { where } i \in\{8,10,11\} .
$$

Thus, assume that $p_{7} p_{9}$ is present. Similarly, assume that $p_{5} p_{7}$ is present, otherwise

$$
\delta\left(p_{7}, p_{5}\right) \geq \frac{|\rho(7, i, 5)|}{\left|p_{7} p_{5}\right|} \geq 1+\sqrt{3}, \text { where } i \in\{3,5,6\} .
$$

Now, as $p_{0} p_{7}, p_{5} p_{7}$ and $p_{7} p_{9}$ are present, $\operatorname{deg}\left(p_{7}\right)=3$. In this case,

$$
\delta\left(p_{7}, p_{8}\right)=\frac{|\rho(7,9,8)|}{\left|p_{7} p_{8}\right|} \geq 1+\sqrt{3}, \text { where } i \in\{5,9\} .
$$

We have thus just shown that $\delta_{0}(P, 3) \geq 1+\sqrt{3}$. For $n \geq 14$, we may assume that $p_{0}=(0,0)$, $p_{3}=(1,0)$, and let $p_{i}=(x+i, 0)$ for $i=13, \ldots, n-1$, where $x \gg 1$ (e.g., setting $x=100$ suffices); finally, let $S=P \cup P^{\prime}$, where $P^{\prime}=\left\{p_{13}, \ldots, p_{n-1}\right\}$. If $u, v \in P \subset S$, then going from $u$ to $v$ via $P^{\prime}$ is inefficient, so as shown earlier in this proof, $\delta(u, v) \geq 1+\sqrt{3}$. Thus, $\delta_{0}(S, 3) \geq 1+\sqrt{3}$, as required. Moreover, this lower bound is tight for both $P$ and $S$; see Fig. 11.(right). 
Remark. If $\Lambda$ is the infinite hexagonal lattice, it is shown in [21] that $\delta_{0}(\Lambda, 3)=1+\sqrt{3}$.

We now continue with degree 4 dilation. We first exhibit a point set $P$ of $n=6$ points with degree 4 dilation $1+\sqrt{(5-\sqrt{5}) / 2}$, and then extend it so to achieve the same lower bound for any larger $n$. Consider the 6 -element point set $P=\left\{p_{0}, \ldots, p_{5}\right\}$, where $p_{1}, \ldots, p_{5}$ are the vertices of a regular pentagon centered at $p_{0}$.

Theorem 3. For every $n \geq 6$, there exists a set $S$ of $n$ points such that

$$
\delta_{0}(S, 4) \geq 1+\sqrt{(5-\sqrt{5}) / 2}=2.1755 \ldots
$$

The inequality is tight for the presented sets.

Proof. Assume that $p_{1}, \ldots, p_{5}$ lie on a circle of unit radius centered at $p_{0}$. Since $\operatorname{deg}\left(p_{0}\right) \leq 4$, there exists a point $p_{i}, 1 \leq i \leq 5$ such that $p_{0} p_{i}$ is not present; we may assume that $i=1$; see Fig. 13 . Observe that

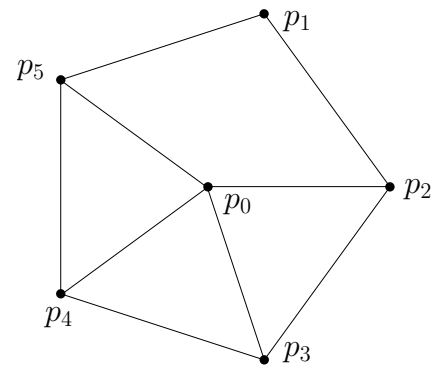

Figure 13: A plane degree 4 geometric graph on the point set $\left\{p_{0}, \ldots, p_{5}\right\}$ that has stretch factor exactly $1+\sqrt{(5-\sqrt{5}) / 2}$, which is achieved by the detour between the pair $p_{0}, p_{1}$.

$$
\left|p_{0} p_{1}\right|=1 \text { and }\left|p_{1} p_{2}\right|=\left|p_{1} p_{5}\right|=\sqrt{1^{2}+1^{2}-2 \cdot 1 \cdot 1 \cos (2 \pi / 5)}=\sqrt{(5-\sqrt{5}) / 2} \text {. }
$$

Now,

$$
\delta\left(p_{0}, p_{1}\right) \geq \frac{|\rho(0, i, 1)|}{\left|p_{0} p_{1}\right|} \geq 1+\sqrt{(5-\sqrt{5}) / 2}=2.1755 \ldots, \text { where } i \in\{2,5\} .
$$

Thus, $\delta_{0}(P, 4) \geq 1+\sqrt{(5-\sqrt{5}) / 2}$. As in the proof of Theorem 2 , the aforesaid six points can be used to obtain the same lower bound for any $n \geq 6$.

To see that the above lower bound is tight, consider the degree 4 geometric graph on $P$ in Fig. 13 whose stretch factor is exactly that, due to the detour between $p_{0}, p_{1}$.

\section{A lower bound on the dilation of the greedy triangulation}

In this section, we present a lower bound on the worst case dilation of the greedy triangulation. Place four points at the vertices of a unit square $U$, and two other points in the exterior of $U$ on the vertical line through the center of $U$ and close to the lower and upper sides of $U$, as shown in Fig. 14 (left). For any small $\varepsilon>0$, the points can be placed so that the resulting stretch factor is at least $\delta\left(p_{0}, p_{3}\right) \geq 2-\varepsilon$. A modification of this idea gives a slightly better lower bound.

Theorem 4. For every $n \geq 6$, there exists a set $S$ of $n$ points such that the stretch factor of the greedy triangulation of $S$ is at least 2.0268 . 
Proof. Replace the unit square by a parallelogram $V$ with two horizontal unit sides, unit height and angle $\alpha \in(\pi / 4, \pi / 2)$ to be determined, as shown in Fig. 14 (right). Place four points at the vertices of $V$ and two other points in the exterior of $V$ on the vertical line through the center of the $V$ and close to the lower and upper side of $V$. First, observe that the greedy triangulation is unique for this point set. Second, observe that there are two candidate detours connecting $p_{0}$ with $p_{3}$ : one of length (slightly longer than) $1+a$ and one of length (slightly longer than) $2 x+b$, where $a$ is the length of the slanted side of $V, b$ is the length of the short diagonal of $V$, and $x$ is the horizontal distance between the upper left corner and the center of $V$.
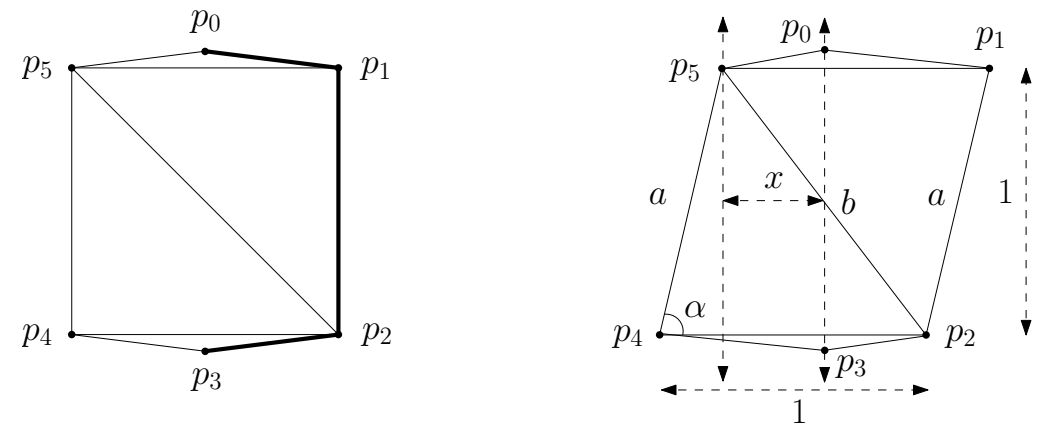

Figure 14: Greedy triangulation of 6 points with stretch factors $2-\varepsilon$ (left) and 2.0268 (right).

A straightforward calculation gives:

$$
\begin{gathered}
a=\frac{1}{\sin \alpha}, b=\frac{\sqrt{1+\sin ^{2} \alpha-2 \sin \alpha \cos \alpha}}{\sin \alpha} \text {, and } x=\frac{1-\cot \alpha}{2} . \\
\text { Let } f(\alpha)=\min \left(1+\frac{1}{\sin \alpha}, 1-\cot \alpha+\frac{\sqrt{1+\sin ^{2} \alpha-2 \sin \alpha \cos \alpha}}{\sin \alpha}\right) \text {, for } \alpha \in\left(\frac{\pi}{4}, \frac{\pi}{2}\right) .
\end{gathered}
$$

Setting $\alpha=1.3416$ (i.e., $\alpha=76.87^{\circ}$ ) yields

$$
\delta\left(p_{0}, p_{3}\right) \geq \max _{\alpha \in(\pi / 4, \pi / 2)} f(\alpha) \geq f(1.3416)=2.0268 \ldots,
$$

as required. As in the proofs of Theorems 2 and 3 , the lower bound can be extended for every $n \geq 6$ in a straightforward way.

\section{Concluding remarks}

In Section 2, we have shown that any plane spanning graph of the vertices of a regular 23-gon requires a stretch factor of $\left(2 \sin \frac{2 \pi}{23}+\sin \frac{8 \pi}{23}\right) / \sin \frac{11 \pi}{23}=1.4308 \ldots$ Henceforth, the question of Bose and Smid [11, Open Problem 1] mentioned in the Introduction can be restated:

Problem 1. Does there exist a point set $S$ in the Euclidean plane such that $\delta_{0}(S)>\left(2 \sin \frac{2 \pi}{23}+\right.$ $\left.\sin \frac{8 \pi}{23}\right) / \sin \frac{11 \pi}{23}=1.4308 \ldots$ ?

Next in Section 3, it has been shown that there exist point sets that require degree 3 dilation $1+\sqrt{3}=2.7321 \ldots$ (Theorem 2 and degree 4 dilation $1+\sqrt{(5-\sqrt{5}) / 2}=2.1755 \ldots$ (Theorem 3 ). Perhaps these lower bounds can be improved. 
Problem 2. Does there exist a point set in the Euclidean plane that has degree 3 dilation greater than $1+\sqrt{3}$ ? Does there exist a point set in the Euclidean plane that has degree 4 dilation greater than $1+\sqrt{(5-\sqrt{5}) / 2}$ ?

Finally in Section 4, we show that the stretch factor of the greedy triangulation is at least 2.0268, in the worst case. Perhaps this lower bound is not far from the truth. Using a computer program we have generated 1000 random uniformly distributed $n$-element point sets in a unit square for every $n$ in the range $4 \leq n \leq 250$, and computed the greedy triangulations and corresponding stretch factors. The highest stretch factor among these was only 1.97 (as attained for a 168-element point set), and so this suggests the following.

Problem 3. Is the worst case stretch factor of the greedy triangulation attained by points in convex position?

Observe that the point set used in the lower bound construction in Theorem 4 is convex, so it is natural to ask: given a non-convex point set $S$ and a greedy triangulation of $S$ having stretch factor $\Delta$, does there always exist a convex subset $S^{\prime} \subset S$ such that the stretch factor of a greedy triangulation for $S^{\prime}$ also equals $\Delta$ ? The point set $S=\left\{p_{1}, \ldots, p_{6}\right\}$ illustrated in Fig. 15 shows that this is not the case. It is routine to verify that the stretch factor of the greedy triangulation of each convex subset $S^{\prime} \subset S$ is at most $1.4753 \ldots<\Delta=1.4772 \ldots$
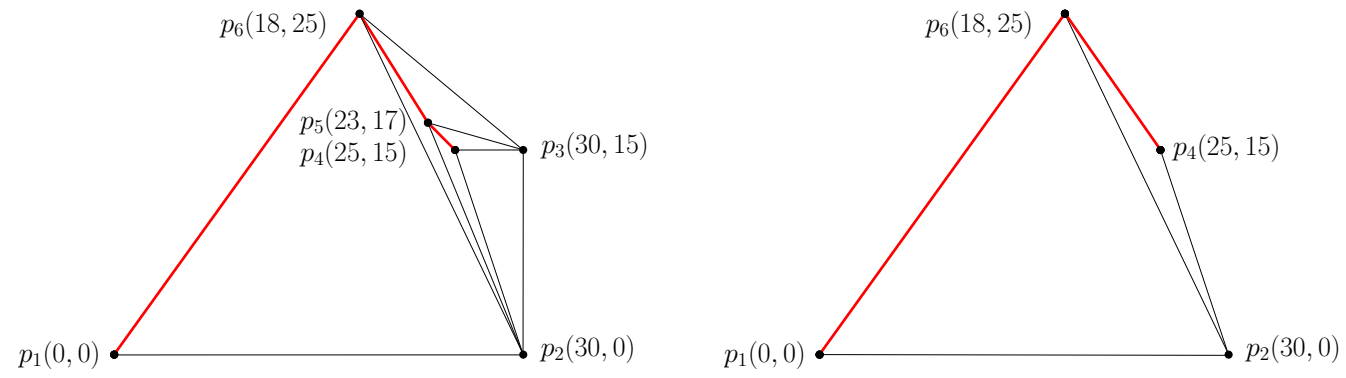

Figure 15: Left: greedy triangulation of a set of 6 points not in convex position with stretch factor $\Delta=$ $1.4772 \ldots$ attained by the pair $\left\{p_{1}, p_{4}\right\}$. Right: the largest stretch factor of the greedy triangulation of a convex subset is that for the subset $S^{\prime}=\left\{p_{1}, p_{2}, p_{4}, p_{6}\right\}$; it is attained by the same pair $\left\{p_{1}, p_{4}\right\}$ and equals $1.4753 \ldots<\Delta$. The corresponding shortest paths are drawn in red color.

Acknowledgment. We convey our special thanks to an anonymous reviewer for suggesting an elegant simplification of the case analysis in the proof of Theorem 1. We express our satisfaction with the software package JSXGraph, Dynamic Mathematics with JavaScript and the OpenMP API, used in our experiments.

\section{References}

[1] P. K. Agarwal, R. Klein, C. Knauer, S. Langerman, P. Morin, M. Sharir, and M. Soss, Computing the detour and spanning ratio of paths, trees, and cycles in $2 \mathrm{D}$ and $3 \mathrm{D}$, Discrete Comput. Geom. 39(1-3) (2008), 17-37.

[2] I. Althöfer, G. Das, D. P. Dobkin, D. Joseph, and J. Soares, On sparse spanners of weighted graphs, Discrete Comput. Geom. 9 (1993), 81-100. 
[3] N. Amarnadh and P. Mitra, Upper bound on dilation of triangulations of cyclic polygons, In Proc. Conf. Comput. Science and Appl., 2006, Springer, pp. 1-9.

[4] B. Aronov, M. de Berg, O. Cheong, J. Gudmundsson, H. J. Haverkort, and A. Vigneron, Sparse geometric graphs with small dilation, Comput. Geom. 40(3) (2008), 207-219.

[5] N. Bonichon, C. Gavoille, N. Hanusse, and L. Perković, Plane spanners of maximum degree six, In Proc. Internat. Colloq. Automata, Lang. and Prog., Springer, 2010, pp. 19-30.

[6] N. Bonichon, I. Kanj, L. Perković, and G. Xia, There are plane spanners of degree 4 and moderate stretch factor, Discrete Comput. Geom. 53(3) (2015), 514-546.

[7] P. Bose, P. Carmi, and L. Chaitman-Yerushalmi, On bounded degree plane strong geometric spanners, J. Discrete Algorithms 15 (2012), 16-31.

[8] P. Bose, L. Devroye, M. Löffler, J. Snoeyink, and V. Verma, Almost all Delaunay triangulations have stretch factor greater than $\pi / 2$, Comput. Geom. 44(2) (2011), 121-127.

[9] P. Bose, J. Gudmundsson, and M. Smid, Constructing plane spanners of bounded degree and low weight, Algorithmica 42 (2005), 249-264.

[10] P. Bose, A. Lee, and M. Smid, On generalized diamond spanners, in Proc. 10th Internat. Workshop on Algorithms and Data Structures, vol 4619 of LNCS, Springer, 2007, pp. 325-336.

[11] P. Bose and M. Smid, On plane geometric spanners: A survey and open problems, Comput. Geom. 46(7) (2013), 818-830.

[12] P. Bose, M. Smid, and D. Xu, Delaunay and diamond triangulations contain spanners of bounded degree, Internat. J. Comput. Geom. Appl. 19(2) (2009), 119-140.

[13] B. Chandra, G. Das, G. Narasimhan, and J. Soares, New sparseness results on graph spanners, Internat. J. Comput. Geom. Appl. 5 (1995), 125-144.

[14] O. Cheong, H. Herman, and M. Lee, Computing a minimum-dilation spanning tree is NP-hard, Comput. Geom. 41(3) (2008), 188-205.

[15] P. Chew, There are planar graphs almost as good as the complete graph, J. Comput. System Sci. 39(2) (1989), 205-219.

[16] S. Cui, I. Kanj, and G. Xia, On the stretch factor of Delaunay triangulations of points in convex position, Comput. Geom. 44(2) (2011), 104-109.

[17] G. Das and P. Heffernan, Constructing degree-3 spanners with other sparseness properties, Internat. J. Found. Comput. Sci. 7(2) (1996), 121-136.

[18] G. Das and D. Joseph, Which triangulations approximate the complete graph? In Proc. International Sympos. on Optimal Algorithms, vol 401 of LNCS, Springer, 1989, pp. 168-192.

[19] D. P. Dobkin, S. J. Friedman, and K. J. Supowit, Delaunay graphs are almost as good as complete graphs, Discrete Comput. Geom. 5 (1990), 399-407.

[20] A. Dumitrescu, A. Ebbers-Baumann, A. Grüne, R. Klein, and G. Rote, On the geometric dilation of closed curves, graphs, and point sets, Comput. Geom. 36 (2006), 16-38. 
[21] A. Dumitrescu and A. Ghosh, Lattice spanners of low degree, preprint, 2016, arXiv:1602. 04381.v1. A preliminary version in Proc. Internat. Conf. Algor. and Discrete Appl. Math., 2016, vol. 9602 of LNCS, pp. 152-163.

[22] A. Ebbers-Baumann, A. Grüne, and R. Klein, On the geometric dilation of finite point sets, Algorithmica 44 (2006), 137-149.

[23] A. Ebbers-Baumann, R. Klein, E. Langetepe, and A. Lingas, A fast algorithm for approximating the detour of a polygonal chain, Comput. Geom. 27 (2004), 123-134.

[24] D. Eppstein, Spanning trees and spanners, in Handbook of Computational Geometry (J. R. Sack and J. Urrutia, editors), North-Holland, Amsterdam, 2000, pp. 425-461.

[25] J. Gudmundsson and C. Knauer, Dilation and detour in geometric networks, in Handbook on Approximation Algorithms and Metaheuristics, Chap. 52 (T. Gonzalez, editor), Chapman \& Hall/CRC, Boca Raton, 2007.

[26] I. Kanj, Geometric spanners: recent results and open directions, in Proc. 3rd Internat. Conf. Comm. and Inform. Technology, IEEE, 2013, pp. 78-82.

[27] I. Kanj and L. Perković, On geometric spanners of Euclidean and unit disk graphs, in Proc. 25th Annual Sympos. on Theoretical Aspects of Computer Science, Schloss Dagstuhl-LeibnizZentrum für Informatik, 2008, pp. 409-420.

[28] I. Kanj, L. Perković, and D. Türkoğlu, Degree four plane spanners: simpler and better, preprint, 2016, arXiv:1603.03818.v1.

[29] M. Keil and C. A. Gutwin, Classes of graphs which approximate the complete Euclidean graph, Discrete Comput. Geom. 7 (1992), 13-28.

[30] R. Klein, M. Kutz, and R. Penninger, Most finite point sets in the plane have dilation $>1$, Discrete Comput. Geom. 53(1) (2015), 80-106.

[31] C. Knauer and W. Mulzer, An exclusion region for minimum dilation triangulations, in Proc. 21st Europ. Workshop Comput. Geom., Eindhoven, 2005, pp. 33-36.

[32] C. Levcopoulos and A. Lingas, There are planar graphs almost as good as the complete graphs and almost as cheap as minimum spanning trees, Algorithmica 8 (1992), 251-256.

[33] X.Y. Li and Y. Wang, Efficient construction of low weighted bounded degree planar spanner, Internat. J. Comput. Geom. Appl. 14(1-2) (2004), 69-84.

[34] W. Mulzer, Minimum dilation triangulations for the regular n-gon, Masters Thesis, Freie Universität Berlin, 2004.

[35] G. Narasimhan and M. Smid, Geometric Spanner Networks, Cambridge University Press, 2007.

[36] M. T. Parvez, Md. S. Rahman and S.-I. Nakano, Generating all triangulations of plane graphs, J. Graph Algorithms Appl. 15(3) (2011), 457-482.

[37] G. Xia, The stretch factor of the Delaunay triangulation is less than 1.998, SIAM J. Comput., 42(4) (2013), 1620-1659.

[38] G. Xia and L. Zhang, Toward the tight bound of the stretch factor of Delaunay triangulations, In Proc. 23rd Canadian Conf. Comput. Geom. (CCCG), 2011. 


\section{Appendix}

Source code. The following parallel $\mathrm{C}++$ code is written using OpenMP in $\mathrm{C}++11$ (notice the pragma directives present in the code). For the set of $N=23$ points, the program ran for approximately 2 days on a computer with quad core processor. The program was compiled with g++ 4.9.2. Please note that older versions of $\mathrm{g}^{++}$might have issues with OpenMP support. Following is a correct way of compiling the program.

$$
\text { g++ program.cpp -std=c++11 -fopenmp -03 }
$$

The number of threads has been set to 4 using the variable numberOfThreads in main(). The user may alter the value of the variable depending on the processor.

Following is the output from the program.

Execution started...

Triangulations checked: 24466267020

Dilation: 1.4308143191

Time taken: 162829 seconds

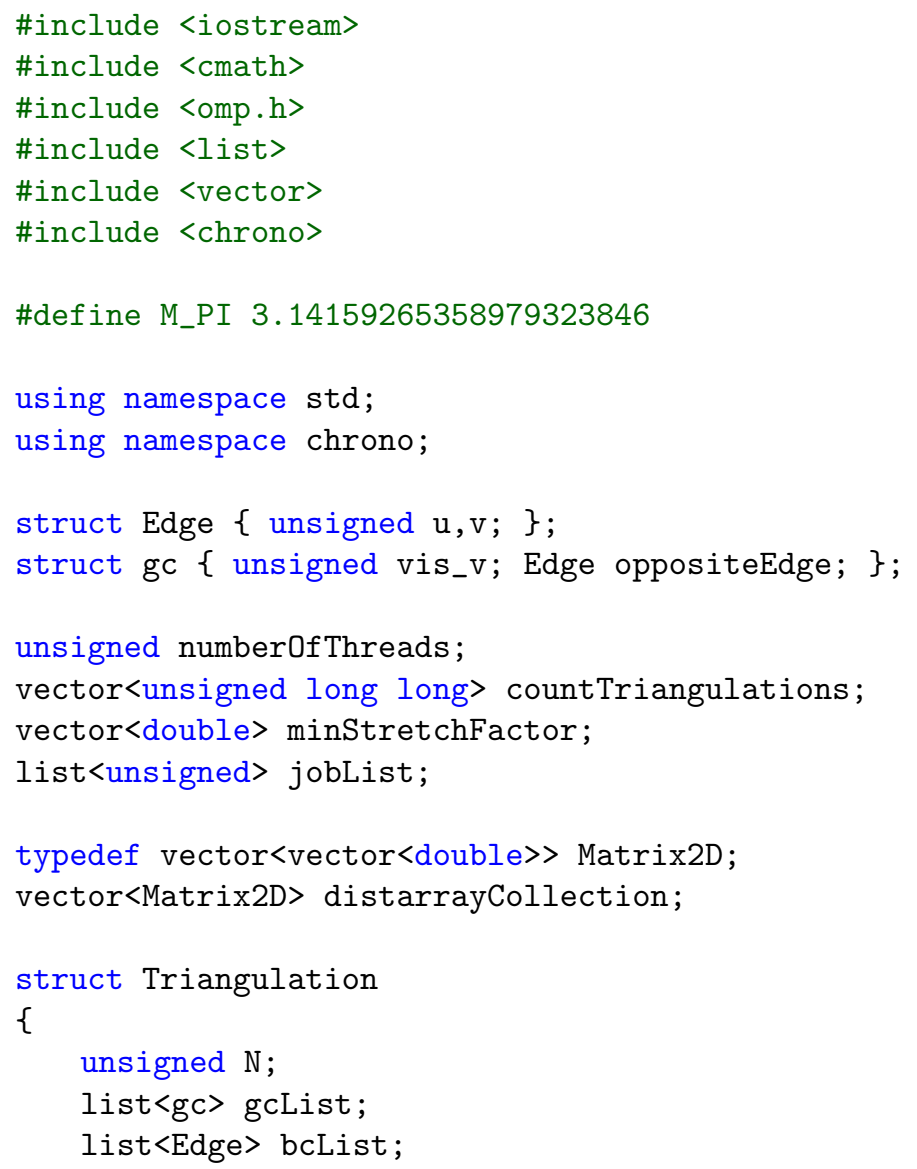




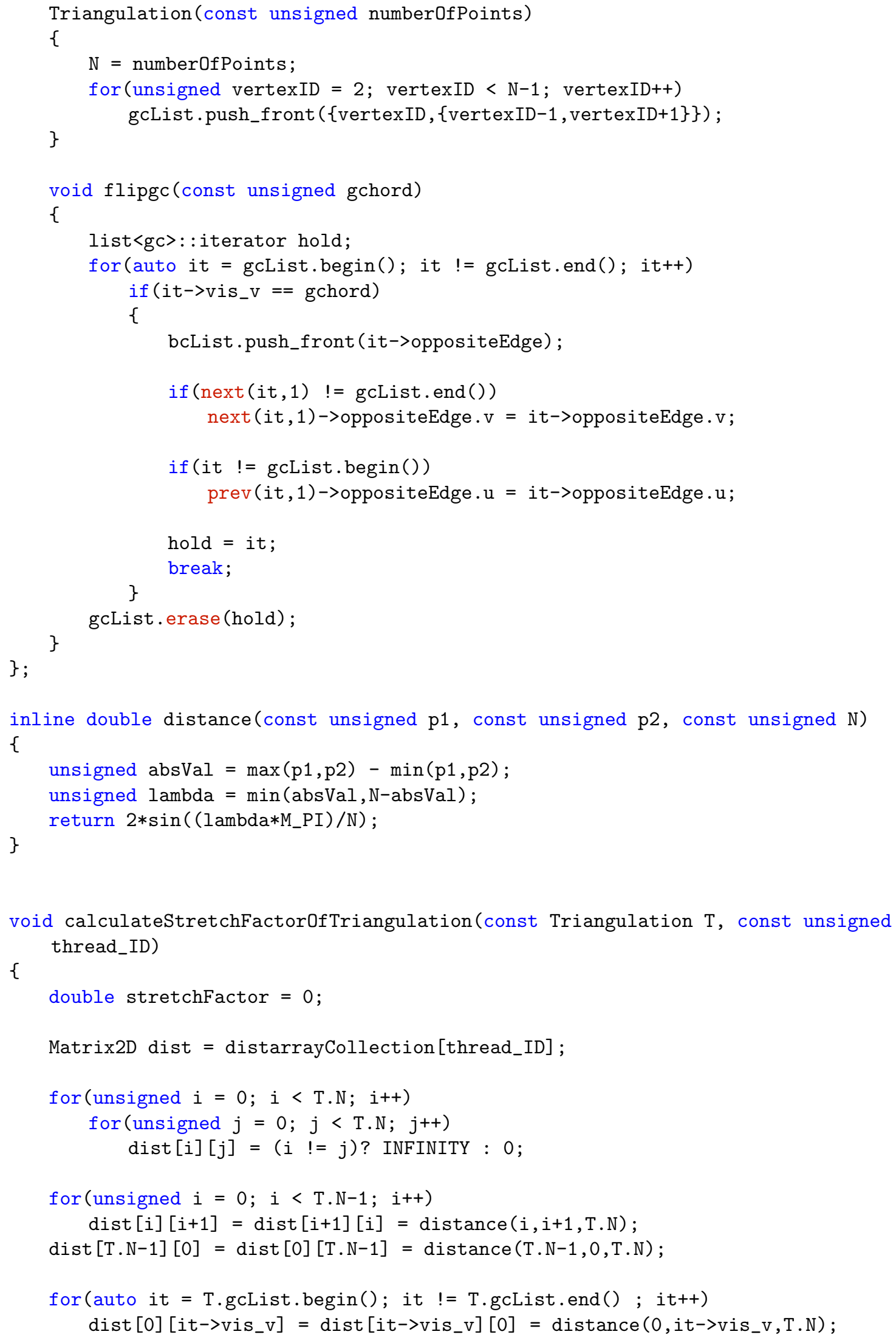




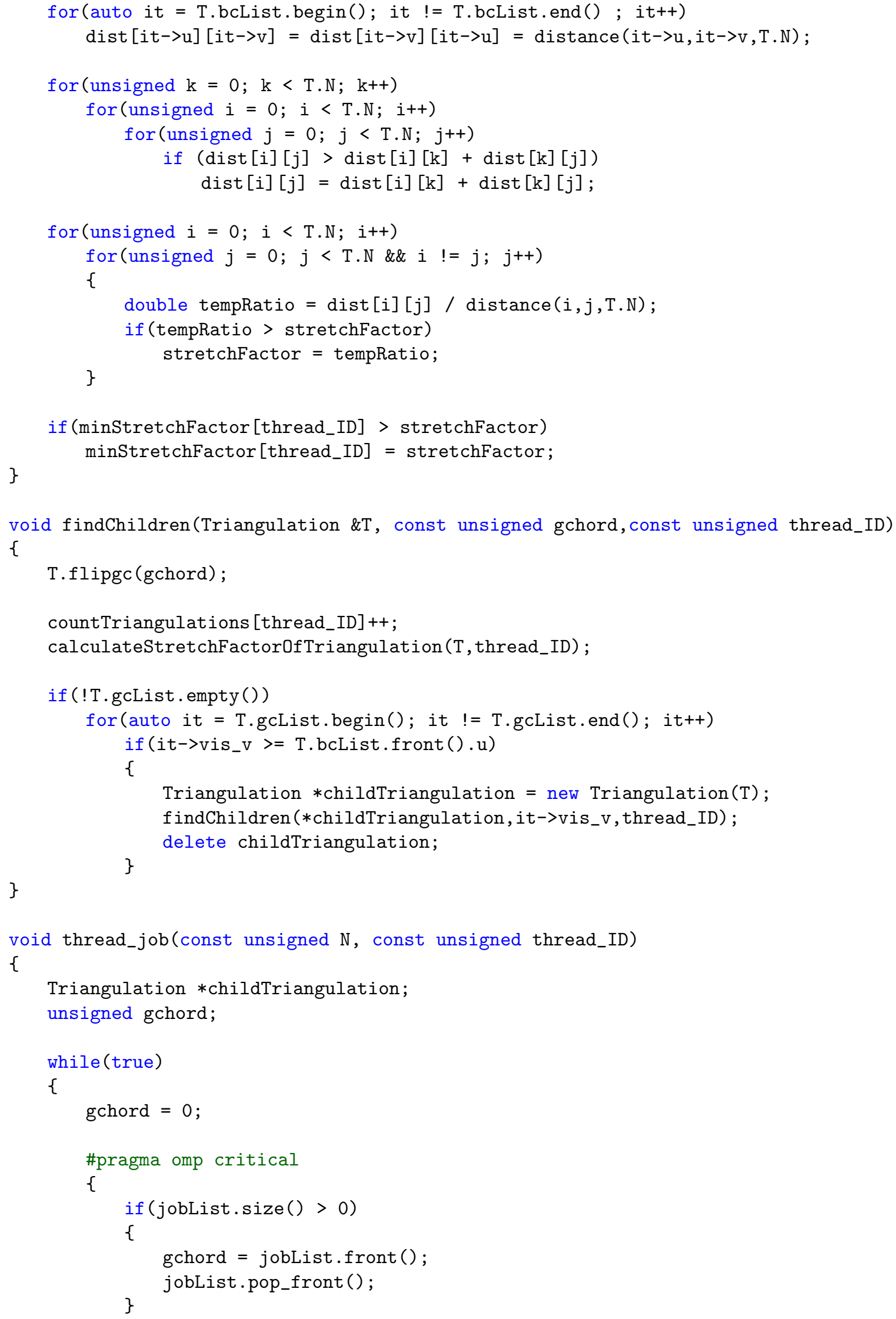




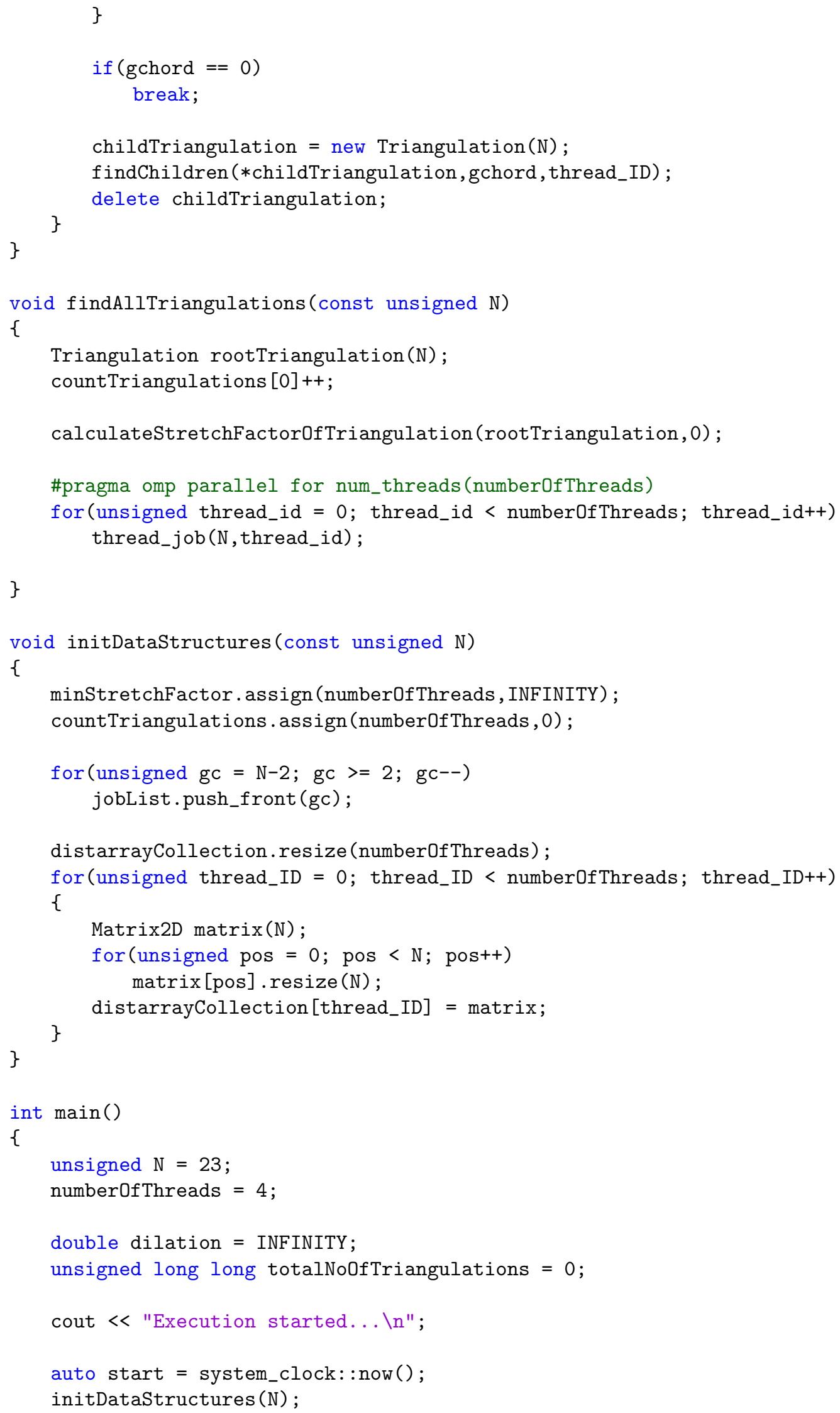




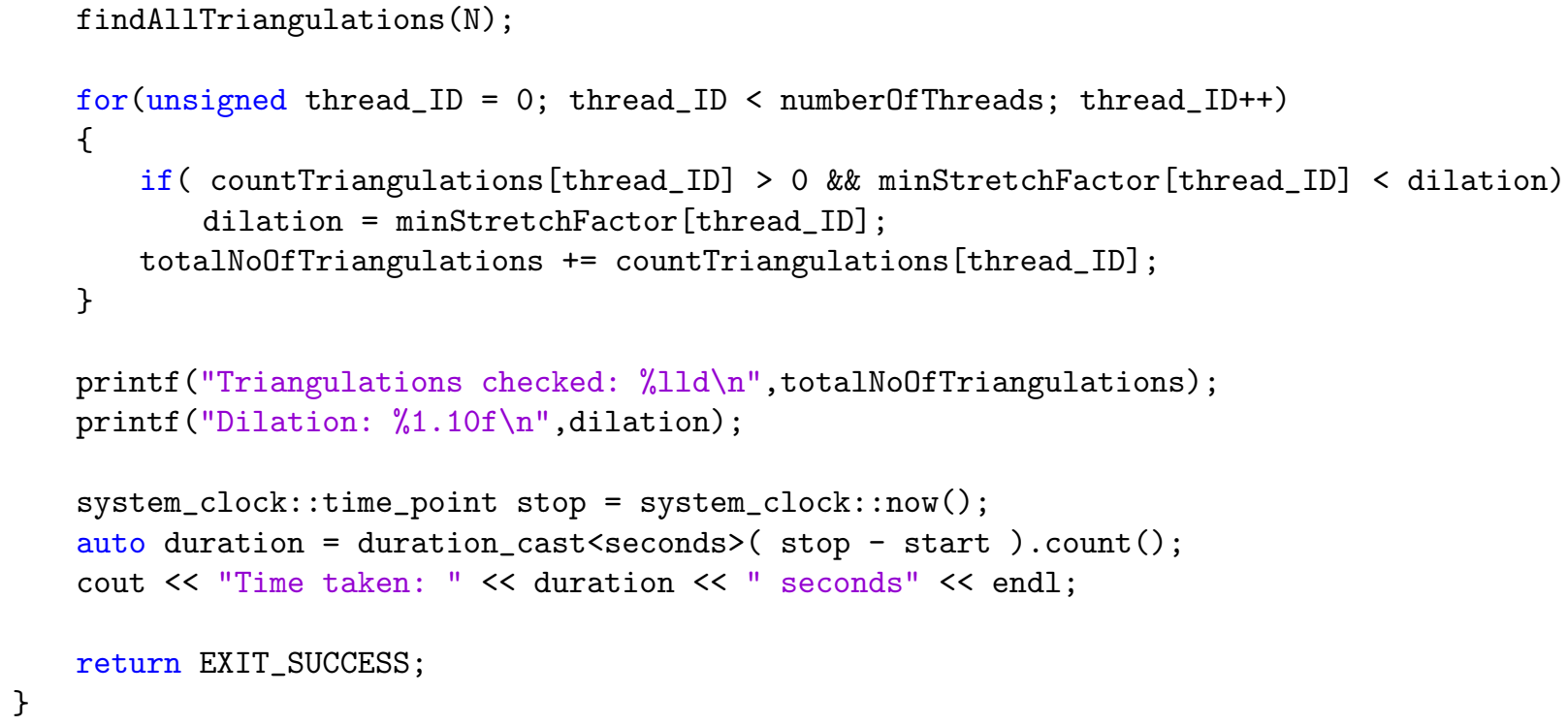

